\title{
Micellar nanocomplexes for biomagnetic delivery of intracellular proteins to dictate axon formation during neuronal development
}

\author{
Giulia Suarato ${ }^{1}$, Seong-Il Lee ${ }^{2}$, Weiyi $\mathrm{Li}^{1}$, Sneha $\mathrm{Rao}^{2}$, Tanvir Khan ${ }^{2}$, \\ Yizhi Meng ${ }^{1}$, and Maya Shelly ${ }^{2 *}$
}

${ }^{1}$ Department of Materials Science and Engineering, Stony Brook University, Stony Brook, NY, USA

${ }^{2}$ Department of Neurobiology and Behavior, Stony Brook University, Stony Brook, NY, USA

* Communicating author: Maya Shelly. E-mail: maya.shelly@stonybrook.edu

(C) 2016. This manuscript version is made available under the Elsevier user license http://www.elsevier.com/open-access/userlicense/1.0/ 


\begin{abstract}
During mammalian embryonic development, neurons polarize to create distinct cellular compartments of axon and dendrite that inherently differ in form and function, providing the foundation for directional signaling in the nervous system. Polarization results from spatiotemporal segregation of specific proteins' activities to discrete regions of the neuron to dictate axonal vs. dendritic fate. We aim to manipulate axon formation by directed subcellular localization of crucial intracellular protein function. Here we report critical steps toward the development of a nanotechnology for localized subcellular introduction and retention of an intracellular kinase, LKB1, crucial regulator of axon formation. This nanotechnology will spatially manipulate LKB1linked biomagnetic nanocomplexes (LKB1-NCs) in developing rodent neurons in culture and in vivo. We created a supramolecular assembly for LKB1 rapid neuronal uptake and prolonged cytoplasmic stability. LKB1-NCs retained kinase activity and phosphorylated downstream targets. NCs were successfully delivered to cultured embryonic hippocampal neurons, and were stable in the cytoplasm for 2 days, sufficient time for axon formation. Importantly, LKB1-NCs promoted axon formation in these neurons, representing unique proof of concept for the sufficiency of intracellular protein function in dictating a central developmental event. Lastly, we established NC delivery into cortical progenitors in live rat embryonic brain in utero. Our nanotechnology provides a viable platform for spatial manipulation of intracellular protein-activity, to dictate central events during neuronal development.
\end{abstract}

Keywords: biomagnetic nanotechnology, chitosan micelles, magnetic nanoparticles, hippocampal neurons, axon development, LKB1 kinase.

Introduction 


\section{Introduction}

During mammalian embryonic development, neuronal cells polarize to create morphologically and functionally distinct compartments of axon and dendrite. Axons and dendrites inherently differ in molecular composition of their cytoplasm, cytoskeleton, and plasma membrane, in morphology and function, to underlie the directed information flow in the mature brain [1-7]. During neuronal development, the polarized architecture of axon and dendrite arises from highly regulated spatial segregation of specific proteins' activities to discrete regions of the cell, to respectively dictate the axonal or dendritic fate $[1-5,8-26]$. Aberrations in the localization of these proteins' activity lead to defective neuron polarization, and underlie developmental neuropathologies including intellectual and motor disabilities, epilepsy and autism spectrum disorders. The ability to exert spatio-temporal control on intracellular protein activity would allow directed regulation of neuronal polarization, and would lead to the generation of new protein-based therapeutic approaches for the repair of neuropathologies that result from break-down of polarity.

Current methodologies for spatio-temporal manipulation of protein function predominantly use light-controlled activation [27-30]. Such approaches were applied to short-term (minutes) localized control of cytoskeleton dynamics by small GTPases, resulting in directed cell protrusions and movement [27] in cultured fibroblasts. However, light-controlled protein activation does not enable its long-term spatial confinement, as the protein can diffuse or actively relocate following activation. Furthermore, molecular-genetics techniques only allow whole-cell up- or downregulation but not spatio-temporal manipulation of protein function. Thus, the ability for spatial restriction of intracellular protein activity to dictate a complex and long-term developmental process as axon formation, which lasts for hours and days, is currently missing. We sought to develop a biomagnetic-based nanotechnology that would deliver and restrict the activity of critical 
intracellular proteins in a spatio-temporal manner to control axon formation. In this study we report the essential steps toward the development of this nanotechnology. We engineered biomagnetic micellar nanoparticles for the purpose of subcellular delivery of a crucial intracellular protein, the kinase LKB1, in developing neurons in culture and the rodent embryonic brain, to dictate axon formation.

Much of the current understanding of the molecular events underlying axon initiation is based on studies using dissociated rodent embryonic hippocampal neurons in culture [1-5]. These neurons undergo polarization - from a cell exhibiting several morphologically similar neurites within hours of plating, to a mature neuron with a single axon and multiple dendrites within days. Studies from these cultured neurons implicated several signaling determinants in axon formation, including the six Par (partitioning-defective) proteins (Par-1 through Par-6) [8, 31]. Recent studies $[20,22,23,25]$ have established the mammalian kinase LsKB1, Par-4 counterpart, as a crucial upstream regulator of axon formation. We and others have shown that down-regulation of LKB1 in cultured hippocampal neurons greatly reduced axon formation [22, 23, 25]. Moreover, deletion of LKB1 in cortical progenitors in the rodent embryonic brain, resulted in striking absence of axon formation in neurons throughout cortical layers in vivo [22, 23, 25]. Furthermore, our previous findings underscore the critical importance for spatial restriction of LKB1-activity in the undifferentiated neurite to promote its axon development, in vitro and in vivo [20,25]. Localized LKB1-activity in a single neurite may initiate axon development via phosphorylation and activation of the down-stream effectors SAD [22, 23] and MARK [17, 32-35] kinases, and their downstream actions on local regulation of the cytoskeleton. SAD-A and SAD-B were shown, in an LKB1-dependent manner, to phosphorylate the axonal microtubule binding protein Tau [22, 23], which regulates local microtubule (MT) organization that is required for axon formation. 
Together, these findings suggest that localized LKB1-activity might serve an instructive signal for axon formation from a single undifferentiated neurite. However, direct demonstration that localized LKB1 activity indeed instructs axon formation, is currently absent. Our overall objective is to develop a biomagnetic-based nanotechnology to control intracellular protein activity, the LKB1 kinase, in a spatio-temporal manner, with the specific goal of locally activating the cell-signaling for axon formation, sustained over a period of $48 \mathrm{hr}$, in culture and in the embryonic brain in vivo. We will direct localized subcellular introduction and retention of LKB1 into single undifferentiated neuritic processes, by spatially manipulating magnetic nanoparticles (MNPs) complexed with LKB1, using an externally applied magnetic field, to dictate the axon fate of these neuritic processes. Continuous localized LKB1-activity delivered in this manner will manipulate axon formation in developing rodent neurons in culture and the embryonic brain.

Here we report the critical steps toward the development of this nanotechnology. First, we created a supramolecular micellar assembly for effectively complexing LKB1 with MNPs to allow its rapid neuronal uptake and prolonged cytoplasmic availability and stability. We show that LKB1 linked to the nanocomplex retained its kinase activity and phosphorylated downstream targets. The nanocomplexes were successfully delivered to cultured embryonic hippocampal neurons and did not affect their growth or development. Importantly, delivery of LKB1-linked nanocomplexes promoted axon formation in these cultured neurons. Lastly, we established a nanotechnology for micellar delivery in cortical progenitor cells in the live rat embryonic brain in utero.

Together, here we report the generation of a multifunctional nanocomplex and show that it is indeed a suitable platform for the localized delivery of LKB1 into an undifferentiated neurite to dictate axonal fate. We emphasize two particularly unique findings reported in this study. First, the demonstration of the promotion of axon formation in cultured hippocampal neurons upon 
delivery of a critical intracellular protein, the kinase LKB1, represents an important proof of concept for the sufficiency of intracellular protein function in dictating a central developmental event as axon formation, over a physiological time scale (2-3 days). Second, the methodology for the intracellular micelle delivery into the live rodent embryonic brain in utero, with the purpose of manipulating neuronal development, represents a unique approach that was established in this study. These findings offer a framework for our overall goal of directing localization of intracellular protein-activity during crucial events of neuronal development.

\section{Methods}

\subsection{Materials and antibodies}

Glycol chitosan (250 kDa molecular weight, degree of deacetylation $>60 \%)$, 5 $\beta$-cholanic acid, N-(3-dimethylaminopropyl)-N'-ethylcarbodiimide hydrochloride (EDC), and N-HydroxySuccinimide (NHS) were purchased from Sigma-Aldrich (St. Louis, MO). Monoreactive hydroxysuccinimide ester of Cyanine 5.5 (Cy5.5-NHS) was obtained from Lumiprobe (Hallandale Beach, FL). Biotin-4-fluorescein (B4F) was obtained from Biotium (Hayward, CA). Superparamagnetic iron oxide nanoparticles (nano-screenMAG-ARA, nano-screenMAGStreptavidin) were purchased from Chemicell (Germany). These magnetic nanoparticles (MNPs) consist of a magnetite core covered by a fluorescence layer $\left(\lambda_{\mathrm{em}, \max }=413 \mathrm{~nm}\right.$ or $\left.613 \mathrm{~nm}\right)$. AntiLKB1 and SAD-antibodies were from EMD Millipore (Billerica, MA). Anti-FLAG®M2 antibody was from Sigma (St. Louis, MO). Neuronal Class III $\beta$-Tubulin (Tuj1) antibody and pan-axonal neurofilament marker SMI-312, were purchased from Covance (Princeton, NJ). Anti-STRAD, anti-Tau, and phospho-site-specific antibody for Tau at Ser-262, were purchased from Santa Cruz Biotechnology (Santa Cruz, CA). Anti- $\beta$-actin antibody was from Cell Signaling Technology 
(Danvers, MA).

\subsection{Plasmid cDNA constructs}

The cDNAs encoding rat LKB1 and STRAD $\alpha$, cloned into the mammalian pcDNA3 expression vector, were previously described [25]. The LKB1 was conjugated to Flag-Tag at the $\mathrm{N}$-terminus, or fused to dTomato (dTom) at the N-ter. To avoid possible effects of dTom fusion on LKB1-activity, we also conjugated $\mathrm{Cy} 3$ fluorophore at the C-ter. cDNAs encoding rat SAD-A and Tau were isolated from total RNA of E18 rat hippocampus by RT-PCR, using oligonucleotide primers (5'-ATGTCGTCCGGGTCCAAGGAA-3' and 5'-TCAGGGTAGAGGGGTTCCATT3') corresponding to SAD-A (GenBank NM_001127337.1) and (5'ATGGCTGAACCCCGCCAGGAG-3' and 5'-TCACAAACCCTGCTTGGCCAAAGA-3') corresponding to Tau (GenBank NM_017212.2), and cloned into pcDNA3 vector.

\subsection{Synthesis of hydrophobically modified glycol chitosan (HGC)}

In order to deliver the kinase LKB1, our protein of interest, a series of synthesis steps were conducted to yield a self-assembled nanocomplex based on the functionalization of chitosan micelles (Fig. 1). First, sulfo-NHS-LC-biotin was conjugated to GC through the formation of amide linkages at the primary amines of the D-gluocosamine residues. Subsequently, biotinylated glycol chitosan (BGC) was allowed to bind with avidin to yield avidinylated BGC (AB-GC). A cholesterol-derivative, $5 \beta$-cholanic acid, was conjugated to AB-GC to yield a self-assembled micellar structure (AB-HGC). Details of the hydrophobic modification step are reported elsewhere [36]. The same hydrophobic modification was also applied to biotinylated glycol chitosan (BGC) 
to yield biotinylated-HGC (B-HGC) for subsequent linking to streptavidin-coated magnetic nanoparticles.

\subsection{Biotinylation of LKB1}

Rat recombinant Flag-tagged LKB1 was generated in human Embryonic Kidney 293T (HEK-293T) cells for preservation of structure and post-translational modifications, to allow optimal function (Fig. 1, immunoblot). Flag-tagged LKB1 was used because of the efficiency of Flag-antibody in LKB1 purification and detection. Flag-tagged LKB1 was purified from HEK293T cell-lysates by affinity purification using Flag-antibody. In a $15 \mathrm{~mL}$ Falcon tube, $3 \mathrm{mg}$ of sulfo-NHS-LC-biotin was suspended in $6 \mathrm{~mL}$ of water to reach a working concentration of 500 $\mu \mathrm{g} / \mathrm{mL}$. A $1 \mathrm{~mL}$ aliquot was added to $1.6 \mathrm{~mL}$ of LKB1 lysate and $0.4 \mathrm{~mL}$ of HPLC water in a 20 $\mathrm{mL}$ scintillation vial. The mixture was incubated for 3 hours at room temperature, under magnetic stirring, before being dialyzed (3.5 kDa MWCO; ThermoScientific) for 2 days against HPLC water to remove unbound biotin. The biotinylated-LKB1 retentate was immediately added to HGC nanocomplexes (see below).

\subsection{Nanocomplex formation}

To generate the Cy5.5-LKB1-chitosan nanocomplex, HGC was complexed with biotinylated-LKB1 via biotin-avidin binding after first being labeled with a fluorophore (Cy5.5; $\lambda_{\mathrm{ex}}=673 \mathrm{~nm}, \lambda_{\mathrm{em}}=707 \mathrm{~nm}$ ) (Fig. 1). Specifically, a $2 \mathrm{~mL}$ suspension of avidinylated-biotinylatedHGC (AB-HGC) $(1.5 \mathrm{mg} / \mathrm{mL}$, in dimethyl sulfoxide, DMSO) was stirred overnight until homogenous. A $6 \mu \mathrm{L}$ volume of Cy5.5-NHS solution ( $5 \mathrm{mg} / \mathrm{mL}$, in DMSO) was added dropwise to $\mathrm{AB}-\mathrm{HGC}$ and the mixture was stirred for 6 hours in darkness at room temperature. The activated 
NHS-ester of the cyanine dye reacts with the free amine on the main polymeric chain of HGC to form an amide linkage. After dialysis for 2 days against HPLC water in dialysis cassettes (10 kDa MWCO; ThermoScientific), the retentate was collected. Complexation of Cy5.5-HGC with LKB1 (Fig. 1) was performed by adding $2.4 \mathrm{~mL}$ of biotinylated-LKB1 (prepared as described above) dropwise to $5 \mathrm{~mL}$ of Cy5.5-AB-HGC $(0.6 \mathrm{mg} / \mathrm{mL})$ and stirring the mixture for 2 hours in darkness at room temperature. The resulting mixture was transferred to Amicon filter tubes $(10 \mathrm{kDa}$ MWCO) and centrifuged at 13,000 $\mathrm{g} g$ for 3 minutes. Centrifugation was repeated 2-3 times until a final retentate volume of $250 \mu \mathrm{L}$ was obtained.

To generate the LKB1-MNP-HGC nanocomplex, biotinylated-HGC (B-HGC) was first complexed with streptavidin-coated magnetic nanoparticles (MNPs) as follows. A suspension of B-HGC $(0.5 \mathrm{mg} / \mathrm{mL}$, in water) was stirred at room temperature until homogenous. A $50 \mu \mathrm{L}$ aliquot of a $10 \mathrm{mg} / \mathrm{mL}$ suspension (in PBS) of $100 \mathrm{~nm}$ streptavidin-coated MNPs (nano-screenMAGStreptavidin; Chemicell) was added to the B-HGC to yield the biotinylated-HGC-MNP (B-HGCMNP) complex (Fig. 1). This mixture was incubated on a rocking platform for 2 hours in darkness at room temperature and probe-sonicated 3 times at 2 minutes each and filtered with a $0.8 \mu \mathrm{m}$ syringe filter. About $0.6 \mathrm{~mL}$ biotinylated-LKB1 was added dropwise to a $2 \mathrm{~mL}$ suspension of BHGC-MNP $(0.5 \mathrm{mg} / \mathrm{mL}$, in water) and the mixture was stirred for 2 hours in darkness at room temperature. The resulting suspension of LKB1-MNP-HGC nanocomplexes (Fig. 1) was collected for material characterization (see section 2.6 below).

\subsection{Material characterization}

Particle size distribution was investigated using Dynamic Light Scattering (NanoDLS Particle Size Analyzer, Brookhaven Instruments Corporation, Holtsville, NY). Specifically, probe- 
sonicated and syringe filtered suspensions of Cy5.5-HGC, MNPs, B-HGC-MNP, Cy5.5-LKB1chitosan, and LKB1-MNP-HGC were sonicated in a water bath (Branson, M2800, Danbury, CT) for 10 min immediately prior to analysis. The particle size measurements were carried out in backscattering mode in triplicate at room temperature.

For transmission electron microscopy (TEM), particles were deposited onto 300-mesh copper TEM grids coated with a Lacey film and air dried. Transmission electron micrographs were captured on a JEOL 1400 TEM (Peabody, MA) using an accelerating voltage of $60 \mathrm{keV}$.

To estimate the number of biotin molecules that were conjugated to $\mathrm{GC}$, a colorimetric assay was used. Specifically, $20 \mu \mathrm{L}$ of the 4'-hydroxyazonenzene-2-carboxylic acid (HABA)/avidin premix solution was added to a 96-wells multi-well plate with $160 \mu \mathrm{L}$ of PBS, and incubated on an orbital shaker. The absorption of the premix solution at $500 \mathrm{~nm}\left(\mathrm{~A}_{500}\right)$ was measured with a spectrophotometric reader (Tecan Infinite ${ }^{\circledR} 200$ PRO, Tecan Trading AG, Switzerland). A $20 \mu \mathrm{L}$ aliquot of BGC (1 mg/mL, in PBS) was then added and the absorption of the mixture at $500 \mathrm{~nm}$ was measured again. Because biotin has a higher affinity for avidin and would displace HABA, the absorption of the HABA/avidin premix at $500 \mathrm{~nm}$ decreased proportionally to the number of biotin molecules. The biotinylation efficiency was defined as the molar ratio of biotins bound on GC to the biotins added in the feedstock. We also performed the HABA assay to quantify the binding efficiency of avidin, by examining the level of biotinylation before and after binding to avidin. This was performed in linear glycol chitosan (GC) polymer only (without hydrophobic modification), to be compatible with the solvents for the HABA assay. 


\subsection{Nanoparticle preparation for cell culture}

Cy5.5-labeled HGC micelles were suspended in serum-free Neurobasal media at a concentration of $0.5 \mathrm{mg} / \mathrm{mL}$ and vortexed for 10 minutes. The suspension was then sonicated with a probe-type sonifier for 3 times ( 2 minutes each, continuously) and again for 2 times ( 5 minutes each, continuously). The system was kept on an ice bath to prevent sudden increases in temperature. Subsequently the suspension was passed through syringe filters (pore sizes $0.8 \mu \mathrm{m}$ and $0.2 \mu \mathrm{m}$; Pall Corporation) as a sterilization method for the following cell culture delivery. MNPs (nano-screenMAG-ARA, Chemicell) were directly diluted in serum-free Neurobasal media to a final concentration of $0.25 \mathrm{mg} / \mathrm{mL}$ and bath sonicated for 10 minutes prior to cell delivery.

To examine the ability of cultured hippocampal neurons to uptake the triple-component chitosan nanocomplex, Cy5.5-labeled HGC was complexed with a model biotinylated moiety, biotinylated-fluorescein (B4F). In this case, LKB1 was not incorporated into the complex to avoid axon formation. Complexation of Cy5.5-HGC with B4F was carried out similarly to the complexation of Cy5.5-LKB1-HGC. Specifically, $100 \mu \mathrm{L}$ of Cy5.5-NHS ester $(5 \mathrm{mg} / \mathrm{mL}$, in DMSO) were added dropwise to $20 \mathrm{~mL}$ of AB-HGC $(2.5 \mathrm{mg} / \mathrm{mL}$, in DMSO $)$ and the mixture was stirred for 6 hours in darkness at room temperature. Afterwards, $50 \mu \mathrm{L}$ of B4F $(10 \mathrm{mg} / \mathrm{mL}$, in DMSO) were added dropwise and the system was stirred for 2 additional hours, in darkness at room temperature. The resulting mixture was transferred to dialysis cassettes (10 kDa MWCO, ThermoScientific) and dialyzed against HPLC water for 72 hours. The retentate consisting of Cy5.5-B4F-HGC was lyophilized and ground into a fine powder. In order to encapsulate the MNPs into the Cy5.5-B4F-HGC nanocomplexes, Pluronic P127 was used as a surfactant. The MNP suspension was diluted in serum-free Neurobasal media containing the resuspended Cy5.5-B4F- 
HGC, in order to obtain a final concentration of $0.125 \mathrm{mg} / \mathrm{mL}$ Cy5.5-B4F-HGC and $0.125 \mathrm{mg} / \mathrm{mL}$ MNPs. The mixture was probe-sonicated for 3 cycles at 2 minutes each and for 2 cycles at 5 minute each. The resulting Cy5.5-B4F-MNP-HGC nanocomplexes were passed through syringe filters (pore sizes $0.8 \mu \mathrm{m}$ and $0.2 \mu \mathrm{m}$; Pall Corporation) as a sterilization method for the following cell culture delivery.

\subsection{Primary hippocampal culture preparation and NCs application}

Cultures of dissociated hippocampal neurons were prepared from embryonic E18 rats as previously described $[2,3]$ in neurobasal medium (Invitrogen, Carlsbad, CA) supplemented with B-27 supplement (Invitrogen). Neurons were plated onto a glass coverslip coated with $100 \mu \mathrm{g} / \mathrm{ml}$ of poly-L-lysine (PLL) and cultured at $37^{\circ} \mathrm{C}$ and $5 \% \mathrm{CO}_{2}$ for the desired time periods. At $2-4 \mathrm{hr}$ following plating, neurons were bath-applied with the NCs at $0.125-0.5 \mathrm{mg} / \mathrm{mL}$, concentrations determined experimentally to allow sufficient NCs uptake for localization in the cytoplasm and neuritic processes. For biocompatibility assays, to examine the normal survival and development of these cultured hippocampal neurons, we bath-applied: (1) Cy5.5-conjugated chitosan micelles alone, (2) MNPs fluorescently labeled with a $413 \mathrm{~nm}$ fluorophore, or (3) triple-component NCs composed of Cy5.5-chitosan micelles, MNPs, and fluorescein. We also tested neuronal survival and development under continued exposure to a $0.08-0.2$ Tesla static magnetic field for $24 \mathrm{hr}$. Untreated neuronal cultures served as control. To determine the promotion of axon formation upon application of LKB1-chitosan NCs, cultures were applied at $4 \mathrm{hr}$ with either LKB1-chitosan-NCs, or control-chitosan micelles at $0.6 \mathrm{mg} / \mathrm{mL}$. We used this slightly higher concentration of NCs than that used for the uptake experiments, to maximize the LKB1 concentration. Neurons were fixed with 4\% formaldehyde in PBS for 10 min, and either directly mounted thereafter, or were co- 
immunostained with the axonal marker Smi-312, together with Tuj-1, a neuron-specific class III $\beta$-tubulin, to determine axon development. For immunolabeling, neurons were permeabilized with $0.1 \%$ Triton $\mathrm{X}-100$, followed by $2 \mathrm{hr}$ blocking with $10 \%$ Normal goat serum (NGS). Immunostaining was carried out in the blocking solution.

\subsection{Examination of NCs localization following uptake}

To directly examine the possible endosomal/lysosomal localization of chitosan NCs in cultured hippocampal neurons, following neuronal attachment, the cells were applied with Cy5.5HGC or LKB1-linked chitosan NCs at a concentration of 100 or $600 \mu \mathrm{g} / \mathrm{ml}$, respectively, for $1 \mathrm{hr}$, while non-uptaken nanoparticles were washed away. The neurons were incubated for different time periods: up to $18 \mathrm{hr}(0,4,8$, and $18 \mathrm{hr})$ for Cy5.5-HGC, or $60 \mathrm{hr}$ for LKB1-linked chitosan NCs. Following incubation, the neurons were fixed with $4 \%$ paraformaldehyde for $10 \mathrm{~min}$, and immunostained with the early endosomal marker EEA1 (BD Bioscience, San Jose, CA) or the lysosomal marker LAMP1 (Enzo Life Sciences, Exeter, United Kingdom). Cells were imaged using a Nikon Super-Resolution Structured Illumination Microscope (SIM).

\subsection{Phenotype classification and neurite length measurements}

Neurons were categorized into three groups of axon differentiation, based on immunostaining with Smi-312. Cells were defined as normally polarized with single axon, if the longest process was 2 times longer than other processes. Neurons harboring more than two processes that showed Smi-312 labeling, were considered as multiple axon cells. Cells specifying no axon were those that contained no processes that were longer than 1.5 times than the other processes, and showed no Smi-312 labeling. These criteria are based on length measurements of 
Smi-312 immunostained neurons. Imaging was performed using confocal laser microscope, with

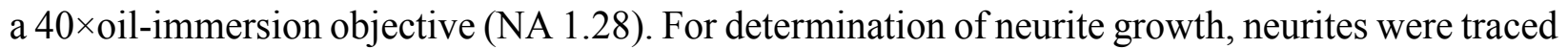
using the ImageJ processing software, and their length determined for each neurite in each cell. Composite tracing of neurites from a random sampling of hippocampal neurons from each treatment, as a measure of neurite length and number, was performed by neurite tracing from the randomly selected cells, and overlaid to generate the composite image.

\subsection{Cell cultures and transfection}

HeLa or HEK-293T cells were grown in DMEM medium supplemented with $10 \%$ FBS, and transiently transfected using calcium-phosphate method. Whole-cell extracts were prepared at $48 \mathrm{hr}$ following transfection for LKB1 purification or the examination of LKB1-kinase activity (see below).

\subsection{Determination of kinase activity of LKB1 linked to chitosan micelles}

To evaluate whether the kinase activity of LKB1 is preserved in the engineered nanocomplexes, and that LKB1-chitosan NCs phosphorylate downstream targets, a biochemical assay was performed using HeLa cells. These cells were used because they do not endogenously express LKB1, SAD-A or SAD-B, whereas they do express the LKB1 co-factors STRAD $\alpha$ and MO25a, essential for LKB1 phosphorylation and promotion of its kinase-activity. Tau was expressed in HeLa cells, either alone, with SAD-A, or in combination with both SAD-A and STRAD $\alpha$, upon transient transfection using calcium-phosphate method. HeLa cell-lysates were prepared $48 \mathrm{hr}$ following transfection and incubated for $2 \mathrm{hr}$ with LKB1-linked-chitosan NCs generated from WT-LKB1, or with control chitosan micelles. Following protein gel 
electrophoresis, Tau phosphorylation was examined by immunoblotting with phospho-sitespecific Ab for Tau at Ser-262, and compared between LKB1-linked chitosan or control-chitosan treated lysates. Immunoblotting with non-phosphorylated Tau Ab served control. Phosphorylation of Tau by the LKB1-chitosan NCs was compared to that of control lysates from cells in which LKB1-cDNA was co-expressed together with Tau, SAD-A and STRAD $\alpha$. Immunoblotting for SAD-A and STRAD $\alpha$ confirmed their expression in the respective lysates. Immunoblotting for $\beta$ actin served control for protein loading.

\subsection{Delivery of chitosan micelles into cortical progenitors in live rat embryonic brain in utero}

Timed-pregnant Sprague Dawley rat at E18 was anesthetized with isoflurane. The abdominal skin was incised longitudinally (about $2-3 \mathrm{~cm}$ ) along the midline and the underlying muscles cut along the linea alba. The uterine horns were exteriorized by laparotomy. Embryos were delicately manipulated to position the ventral side facing up and to expose the cerebral hemispheres under the transparent uterine membrane. Approximately $1 \sim 2 \mu \mathrm{L}$ of chitosan micelles $(0.5 \mathrm{mg} / \mathrm{mL}$ ) labeled with Cy5.5 fluorophore, (together with $0.3 \mathrm{mg} / \mathrm{ml}$ sterile fast green dye), suspended in PBS solution, were injected with a hand-positioned micropipette attached to a pressure-controlled pipette system, into one of the lateral ventricles of the embryos through the uterine membrane. We predicted that micellar delivery into the lateral ventricles, will allow their uptake by a subset of cortical neuronal post-mitotic progenitors lining the ventricular wall, to allow their entry into the neuronal cytoplasm in vivo. Injection at E18 should mostly label layer II/III pyramidal cortical progenitors $[37,38]$, whereby fluorescently-tagged chitosan micelles will allow the fluorescent tracking of these developing and migrating neuronal cells in the embryo. The uterine horns were then returned into the abdominal cavity, the wall and skin sutured, and the 
embryos were allowed to continue their development for 2 days. For cortical neuronal imaging and analysis, E20 brains were dissected and fixed with 4\% paraformaldehyde. Brains were sectioned coronally with a vibratome (Leica, VT1000S) at the level of the somatosensory cortex, and the slices $(80 \mu \mathrm{m})$ were mounted in Fluomount G (Southern Biotech, Inc.). Confocal images (60-80 $\mu$ m-thick z-stacks) of isolated neurons were acquired using Cy5.5 fluorescence with a 40x (N.A. 1.43) oil-immersion objective. A total of $3 \sim 5$ embryonic brains were analyzed from 2 separate timed-pregnant rats. All animal protocols were approved by the Institutional Animal Care and Use Committees of Stony Brook University. Timed pregnant Sprague Dawley rats were purchased from Charles River.

\section{Results}

\subsection{Physicochemical characteristics of LKB1-MNP-chitosan nanocomplexes (LKB1-NCs)}

We describe the design and fabrication (Fig. 1) of biomagnetic micellar LKB1nanocomplexes (LKB1-NCs), assembled using a naturally-derived polymer, chitosan, that serves two crucial purposes. First, chitosan should allow rapid delivery of the NCs into the cell $[39,40]$ (see Fig. 3), and enable their escape from endosomal entrapment and lysosomal degradation [4143] (Supplementary Fig. S3). Nanoparticle capture within the endosomal-lysosomal pathway as a consequence of endocytosis is the predominant barrier for enabling interaction of LKB1 with cytoplasmic targets (see Fig. 3 and 6, and Supplementary Fig. S3). To avert this problem and facilitate LKB1 delivery into neuronal cells, LKB1 (Fig. 1, step 5) and MNPs (Fig. 1, step 4) were separately complexed with chitosan. Second, chitosan is assembled into a micellar structure with a defined size [36, 44] (see Fig. 2), with hydrophilic shell and hydrophobic core, that will serve as scaffold for biotin-avidin $[45,46]$ mediated conjugation of the MNPs and LKB1 on the hydrophilic 
shell (Fig. 1 and 2). In this micellar configuration, LKB1 and MNPs should extend into the cytoplasm (Fig. 1, step 6) where LKB1 would be readily active while locally anchored to the MNPs by the magnetic field. In the aqueous cytoplasm, the chitosan micelle will represent a stable coreshell structure that, together with the strong biotin-avidin linkage, will allow prolonged LKB1 and MNP binding (see Fig. 1, 3 and 6), resulting in sustained LKB1-activity in the neuronal cytoplasm (see Fig. 3, 6, and 7). Each moiety in this NC, LKB1, MNPs, and chitosan, is uniquely fluorescently labeled (see Fig. 1 and 3) to track the integrity and stability of the NC within the cell. We now describe a stepwise assembly of this multifunctional NC and the advantages of our design.

Self-assembled chitosan micelles. In the aqueous environment, chitosan, a linear polymer, self-assembles into micellar nanoparticles upon chemical modification [36, 44]. To assemble these hydrophilic shell-hydrophobic core micelles, we used the water soluble glycol chitosan (GC). The amine groups on the backbone of the GC polymer were conjugated to the cholesterol derivative $5 \beta$-cholanic acid, to yield hydrophobically modified glycol chitosan (HGC), which have the characteristics of an amphiphilic micelle capable of self-assembling into a $200 \mathrm{~nm}$ nanoparticle [36] (Fig. 2A). The dimension of the chitosan micelle is important as it affects its uptake and intracellular routing, and is crucial for localization in the fine neuritic processes, only few microns in diameter. While the hydrophilic shell serves as a scaffold for LKB1 and MNPs linking, the hydrophobic core allows the encapsulation of fluorescence molecules, e.g. Cy5.5 (Fig. 1, step 4a; see Fig. 3, 6 and 7).

Magnetic nanoparticles (MNPS). We used superparamagnetic iron oxide nanoparticles as the MNPs, because they do not have a magnetic moment in the absence of an external magnetic field, allowing optimal "on - off" magnetic manipulation of their localization. We chose streptavidin coated MNPs (100 nm diameter; Fig. 2B) as a coupling agent with biotinylated-HGC, 
yielding B-HGC-MNP nanocomplex (Fig. 1, step 4b and Fig. 2C). The streptavidin coating of the MNPs was also used to link to the biotinylated LKB1 protein (Fig. 1, step 6b; see below). Notably, the MNPs are fluorescently labeled with a $413 \mathrm{~nm}$ (emission) fluorophore. DLS and TEM data confirmed that the MNPs are $100 \mathrm{~nm}$ in diameter (Fig. 2B).

To create the linkage between the HGC micelle and the MNPs, we first conjugated a biotin molecule, sulfo-NHS-LC-biotin, to the free amine groups on the backbone of GC prior to hydrophobic modification (Fig. 1, step 1). The biotinylated-chitosan (BGC), containing linkage points for avidin binding, was self-assembled into a biotinylated-HGC micelle, B-HGC, after hydrophobic modification with $5 \beta$-cholanic acid (Fig. 1, step 3b). Biotin quantitation of BGC using a (4'-hydroxyazobenzene-2-carboxylic acid) (HABA) assay estimated that 15 molecules of biotin were conjugated to each molecule of GC (Table $\mathrm{x}$ ). We also estimated, using the HABA assay, that 2 of these biotin molecules bound to avidin during the avidinylation step (Fig. 1, Table). Biotinylated-HGC was complexed to MNP, yielding a nanoparticle with an average diameter of $168 \mathrm{~nm}$ (Fig. 2C).

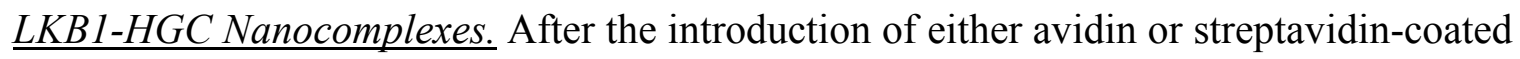
MNPs to the chitosan micelle, the remaining biotin binding sites on the Avidin-Biotin-HGC or Biotin-HGC-MNP were now linked with Flag-tagged or dTomato-fused biotinylated-LKB1, to generate the triple-component $\mathrm{NC}$ (Fig. 1, step 6). Of note, in the biocompatibility assays in neuronal cells, to examine normal neuronal development upon triple-component $\mathrm{NC}$ application (see Fig. 3D,F), we used fluorescein-linked NCs and not LKB1-linked NCs, to avoid LKB1induced axon formation (see Fig. 6). We therefore also linked biotinylated-fluorescein to biotinylated-HGC micelle via avidin binding. Although fluorescein has a lower molecular weight 
than the LKB1 protein, it serves a good substitute for evaluating the stepwise synthesis for the generation of the nanocomplex.

Rat recombinant LKB1 was generated in human Embryonic Kidney 293T (HEK-293T) cells for preservation of structure and post-translational modifications, to allow optimal function (Fig. 1, step 6, immunoblot). We used Flag-tagged LKB1 for a biochemical analysis to determine the kinase activity of NC-linked LKB1 (see Fig. 5), because of the efficiency of Flag-antibody in LKB1 purification and detection. Having examined the kinase activity of Flag-tagged LKB1, it was also used for a functional assay to determine the ability of LKB1-NCs to promote axon formation in rat dissociated cultured embryonic hippocampal neurons (see Fig. 6). For fluorescent tracking of the LKB1 protein, it was fused to dTomato (dTom) at the N-ter, or, to avoid possible effects of dTom fusion on LKB1-activity, we also conjugated Cy3 fluorophore at the C-ter (data not shown).

According to our calculations from the HABA assay (Fig. 1, Table), the molar ratio between the chitosan micelles and LKB1 is estimated to be 13:1 (we used a HGC:LKB1 mass feedstock ratio of 52:1). DLS and TEM findings showed that Cy5.5-LKB1-chitosan (Fig. 2D) and LKB1-MNP-HGC (Fig. 2E) nanocomplexes were $176 \mathrm{~nm}$ and $184 \mathrm{~nm}$ in diameter, respectively. This reduction in size compared to the $200 \mathrm{~nm}$ control chitosan micelles (Fig. 2A), is likely due to a slight compaction of the micellar structure because of hydrophobic interactions between avidin and the micelle core.

\subsection{Uptake, stability, and biocompatibility of NCs in cultured hippocampal neurons}

Next, we examined the uptake, cellular stability and integrity of the NCs in dissociated rat embryonic cultured hippocampal neurons, by fluorescently tracking co-localization between each 
moiety of the NC for a prolonged period of up to $48 \mathrm{hr}$ (Fig. 3 and Supplementary Fig. S1). These analyses also allowed us to determine the biocompatibility of our NCs with the survival and development of these cultured neurons. Cultured hippocampal neurons undergo a wellcharacterized, stereotypical polarization process to establish the distinct compartments of axon and dendrites [2, 3] (Fig. 3A). The polarization process has been divided into five stages. Shortly after culturing, the neuron forms small membranous protrusions (stage 1) that develop into short and morphologically similar neurites $20-30 \mu \mathrm{m}$ in length within 6-12 $\mathrm{hr}$ (stage 2). Apparent polarity first emerges after $24 \mathrm{hr}$ (stage 3), when one of the neurites exhibits accelerated growth and acquires axonal characteristics. Maturation of axon and dendrites occurs in subsequent days (stage 4), followed by synapse formation (stage 5). In these assays, we tested application of each of the NC components alone, as well as the triple-component NC (Fig. 3 and Supplementary Fig. S1). Rat embryonic day 18 (E18) hippocampal neurons were cultured and allowed to adhere for $2 \mathrm{hr}$. The NCs were bath-applied at $0.125-0.5 \mathrm{mg} / \mathrm{mL}$, concentrations determined experimentally to allow sufficient NCs uptake for localization in the cytoplasm and neuritic processes (Fig. 3B,C,D and Supplementary Fig. S1). We bath-applied: (1) Cy5.5-conjugated chitosan micelles alone (Fig. 3B,E and Supplementary Fig. S1A), (2) MNPs fluorescently labeled with a $413 \mathrm{~nm}$ fluorophore (Fig. 3C and Supplementary Fig. S1B), or (3) triple-component NCs composed of Cy5.5-chitosan micelles, MNPs, and fluorescein (Fig. 3D,F). Of note, in the biocompatibility assays for the triplecomponent NCs (Fig. 3D,F), to examine normal neuronal development, we used fluorescein linking and not LKB1-linked NCs, to avoid LKB1-induced axon formation (see Fig. 6). Furthermore, we tested neuronal survival and development under continued exposure to a 0.08 0.2 Tesla static magnetic field for $24 \mathrm{hr}$ (Supplementary Fig. S2), considered to be safe for cultured 
neurons [47], in neurons that were also applied with MNPs. Untreated neuronal cultures served as control.

We observed that chitosan micelles or MNPs alone, or triple-component NCs were uptaken by the neuronal cells within few minutes (5-15 min; data not shown). NCs stability within the cytoplasm was assayed by NCs application for prolonged periods (18-48 hr), as axon formation starts at $12 \mathrm{hr}$ and is completed by $24-48 \mathrm{hr}$ (Fig. 3A). Following neuron fixation, high-resolution confocal images were acquired for the chitosan micelles (Fig. 3B and Supplementary Fig. S1A), MNPs (Fig. 3C and Supplementary Fig. S1B), or the triple-fluorescent components of the NCs (Fig. 3D). In these assays, we avoided immunolabeling with neuronal or axon and dendrite specific markers, as during the permeabilization step for immunolabeling, NCs escape the cytoplasm (see Fig. 6). Neuronal morphology was thus visualized by phase-contrast imaging, which only enabled reliable enumeration of the neuritic processes, but not axon/dendrite determination. Our findings showed that uptaken NCs were present in the cytoplasm and the neuritic processes (Fig. 3B,C,D, arrows, and Supplementary Fig. S1). By examining co-localization between the triple components of the $\mathrm{NC}$, we determined nanocomplex integrity in the cytoplasm for prolonged times, to ensure that chitosan remained complexed to fluorescein and MNPs (Fig. 3D). Our findings showed clear co-localization between fluorescein, MNPs and chitosan $30 \mathrm{hr}$ after delivery (Fig. 3D), indicating that NCs integrity and stability is preserved in the cytoplasm for sufficient time for axon formation.

Together, our findings show that application of chitosan micelles, triple-component NCs, or a static magnetic field did not affect neuronal viability or development (Fig. 3B,C,D and Supplementary Fig. S1 and S2). Composite tracings of neurites from randomly sampled neurons from chitosan micelles (Fig. 3E) or triple-component NCs (Fig. 3F) applied cultures, or cultures applied with MNPs and treated with the magnetic field (Supplementary Fig. S2A,B), confirmed 
the lack of significant effect on neurite growth or number, compared to respective control cultures. Quantitative measurements of average individual or total neuritic length (Fig. 4A,B and Supplementary Fig. S2C), and average neurite number for each cell (Fig. 4C,D and Supplementary Fig. S2D), revealed that no differences were found between control and chitosan- (Fig. 4A,C) or magnetic field-applied neuronal cultures (Supplementary Fig. S2C,D). When the neurons were exposed to the triple-component NCs, there was an observable difference in growth (i.e., neurite length) (Fig. 4B); however, the number of neurites remained the same (Fig. 4D), and there was no visible effect observed on neuronal survival or development (Fig. 3D,F).

To directly determine NCs stability in the neuronal cytoplasm for prolonged periods, we examined the localization of chitosan micelles with the lysosomal marker LAMP1 at different times following uptake (up to $24 \mathrm{hr}$ ) in cultured hippocampal neurons (Supplementary Fig. S3). Micelles were applied at $100 \mu \mathrm{g} / \mathrm{ml}$, a concentration determined experimentally to allow particle resolution, for $1 \mathrm{hr}$, while non-uptaken nanoparticles were washed away. The neurons were then incubated up to $24 \mathrm{hr}(4,8,18$ or $24 \mathrm{hr})$. Cells that were not incubated further following application $(0 \mathrm{hr})$, were considered control. The neurons were imaged using a Super-Resolution Structured Illumination Microscope (SIM). These SIM images demonstrated conclusively the robust nanoparticle uptake by the neurons and the chitosan presence in the soma (supplementary Fig. S3A,B,C) and the neuritic processes (supplementary Fig. S3Bb-d and Ca,b). Despite the extensive presence of chitosan micelles in these neurons at all times examined, at no time did they associate with LAMP1 (Supplementary Fig. S3B,C). Even at late examination time of $18 \mathrm{hr}$, the LAMP1 and chitosan fluorescence signals remained mostly distinct throughout the soma (supplementary Fig. S3C) and the neuritic processes (supplementary Fig. S3Ca,b). We conclude that in these cultured hippocampal neurons the micelles are not localized in lysosomes at any time, suggesting 
that they escape lysosomal degradation, and are likely present in the neuronal cytoplasm, where they appear to be stable for prolonged periods of time (Fig. 3B,D and Supplementary Fig. S3; see also Fig. 6).

Together, these NCs delivery experiments demonstrated that we have established a suitable platform for subcellular delivery of intracellular protein-linked nanocomplexes into cultured hippocampal neurons. We thus next generated the LKB1-linked NCs, and examined their ability in activating downstream targets (Fig. 5), and in the promotion of axon formation in cultured hippocampal neurons (Fig. 6).

\subsection{Kinase activity of LKB1-chitosan nanocomplexes}

Having confirmed that our NC configuration is biocompatible with survival and development of cultured neuronal cells, we next examined whether LKB1-linked chitosan NCs phosphorylate downstream targets by biochemical analysis (Fig. 5). LKB1 phosphorylates and activates SAD-A and SAD-B kinases in the embryonic brain [22, 23], that subsequently phosphorylate the MT-associated protein Tau at Serine residue 262, necessary events in LKB1mediated axon formation $[22,23]$ (Fig. 5A). We assayed whether LKB1-linked chitosan micelles phosphorylated Tau in the presence of SAD-A kinase [22], using HeLa cells (Fig. 5B). These cells were chosen because they do not endogenously express LKB1, SAD-A or SAD-B, whereas they do express the LKB1 co-factors STRAD $\alpha$ and MO25 $\alpha$, essential for LKB1 phosphorylation and promotion of its kinase-activity [48, 49]. We cloned the Tau, SAD-A, and STRAD $\alpha$ cDNAs, from E18 rat hippocampus and prepared mammalian expression vectors of these proteins. Tau was expressed in HeLa cells, either alone, with SAD-A, or in combination with both SAD-A and STRAD $\alpha$ (Fig. 5B). At $48 \mathrm{hr}$ following transfection, HeLa cell-lysates were prepared and 
incubated for $2 \mathrm{hr}$ with LKB1-linked or control-chitosan micelles. Following protein gel electrophoresis, Tau phosphorylation was examined by immunoblotting with phospho-sitespecific antibody (Ab) for Tau at Ser-262, and compared between LKB1-linked NCs or chitosanonly micelles (Fig. 5B). Immunoblotting with non-phosphorylated Tau Ab served control for Tau expression. These experiments demonstrated that LKB1-linked NCs indeed phosphorylated Tau at Ser-262 only in lysates that co-expressed SAD-A and STRAD $\alpha$ together with Tau (Fig. 5B). Phosphorylation of Tau by LKB1-chitosan micelle was comparable to that of control lysates from cells in which LKB1-cDNA was co-expressed together with Tau, SAD-A and STRAD $\alpha$ (Fig. 5B, right). These biochemical analyses demonstrated that in our NC configuration, chitosan-linked LKB1 maintains its kinase activity. We note that in these experiments, there was dependency on the presence of STRAD $\alpha$ protein, an essential cofactor for LKB1-activity $[48,49]$, for LKB1mediated phosphorylation of Tau. However, in the functional assay for LKB1-NCs-induced axon formation in cultured hippocampal neurons (see Fig. 6), LKB1-linked chitosan alone was sufficient to promote axon formation, without the requirement for STRAD $\alpha$ presence. We conclude that the requirement for STRAD $\alpha$ in the biochemical analysis (Fig. 5B) might reflect assay detection sensitivities, whereby STRAD $\alpha$ might promote enhanced LKB1-NCs-activity, compared to its activity alone.

3.4. Promotion of axon formation in cultured hippocampal neurons by LKB1-chitosan nanocomplexes

Having confirmed by biochemical analysis that LKB1-chitosan NCs phosphorylate downstream targets (Fig. 5), we next inquired whether these LKB1-NCs promote axon formation in cultured hippocampal neurons (Fig. 6). Rat embryonic E18 hippocampal neurons were allowed 
to adhere for $4 \mathrm{hr}$, followed by bath application of either LKB1-chitosan-NCs or control-chitosan micelles at $0.6 \mathrm{mg} / \mathrm{ml}$ (Fig. 6). We used this slightly higher concentration of NCs than that used for the uptake experiments (Fig. 3 and 4), to maximize the LKB1 concentration. Axon formation was examined $60 \mathrm{hr}$ following NCs application, sufficient time for axon development, and compared between control-chitosan or LKB1-chitosan treated cultures. Cell morphology and axon formation were examined by co-immunostaining with the axonal specific marker Smi-312, together with Tuj-1, a neuron-specific class III $\beta$-tubulin that labels the entire neuronal cell including soma and neuritic processes, and compared between LKB1-chitosan-NCs and controlchitosan applied cultures (Fig. 6 and Supplementary Fig. S4).

Qualitative observations (Fig. 6A,B and Supplementary Fig. S4) revealed that in control cultures, as expected, most neurons developed only a single axon and multiple dendrites (see Fig. 3A), confirmed by tracings of axon and dendrites from randomly sampled neurons in control cultures, determined based on Smi-312 labeling (Fig. 6B and Supplementary Fig. S4). In stark contrast, in cultures applied with LKB1-chitosan-NCs, many cells formed more than one axon, most of which formed at least 3 axons (Fig. 6A,B and Supplementary Fig. S4), with no effect on the number of dendrites (data not shown). Conservative quantitative measurements of the percentage of cells that formed more than one axon, revealed that whereas in control cultures, less than $10 \%$ of cells formed multiple axons, approximately $25 \%$ of cells formed multiple axons in cultures applied with LKB1-chitosan-NCs (Fig. 6C,D). Furthermore, we observed that the Cy5.5 fluorescence from chitosan, was well localized with that of Tuj-1 in the soma (Fig. 6E), indicating that the LKB1-linked micelles were stable in the cytoplasm for the prolonged time of $60 \mathrm{hr}$, the time of cell examination. Importantly, immunolabeling with the lysosomal marker LAMP1 confirmed that at $60 \mathrm{hr}$, the LKB1-linked chitosan micelles present in these neurons were not 
localized in lysosomes (Fig. 6F), suggesting their likely release from lysosomal degradation and their presence in the neuronal cytoplasm, similar to that observed with control chitosan micelles (Supplementary Fig. S3). We note that some NCs might have escaped the cytoplasm during the permeabilization for immunolabeling, compared to the robust NCs observed in the cytoplasm without permeabilization (see Fig. 3). Together with the biochemical examination (Fig. 5), this functional analysis (Fig. 6) indicates that LKB1, linked to chitosan NCs, maintains kinase activity in phosphorylating downstream targets and, consequently, promotes axon formation in cultured hippocampal neurons.

\subsection{Chitosan micelle delivery to cortical progenitors in a live rat embryonic brain in utero}

Our ultimate goal is to manipulate axon development in the live rodent embryonic cortex. Manipulation of axon formation in cultured neurons and in the developing embryonic brain in vivo, presents fundamentally different challenges. Mechanistically, however, the same molecular determinants and signaling pathways have been shown to dictate axon formation in vitro and in vivo. In particular, we and others have shown that LKB1 is a critical determinant, necessary for axon formation in cortical pyramidal neurons in vivo [22, 23, 25]. In line with our goal of directing axon formation in cortical progenitors in vivo, here we developed a methodology for intracellular micelle delivery into the live rodent embryonic brain in utero. This methodology represents a unique approach that was established in this study (Fig. 7 and Supplementary Fig. S5).

Upon completion of mitosis, cortical progenitors start to migrate radially to populate the different layers of the developing cortex [37, 50-55] (Fig. 7B). During migration, cortical pyramidal neurons undergo stereotypical morphological changes leading to polarized development of axon and dendrite [37] (Fig. 7B). The newborn neuron acquires a bipolar morphology in the 
ventricular zone (VZ), with apical-leading and basal-trailing processes. The trailing process will become the axon and grow towards its target. We and others have identified LKB1 as key regulator of axon formation $[22,23,25]$ in these cortical neurons in vivo. Selective deletion of $l k b 1$ in mouse cortical pyramidal neurons $[22,23]$, or down-regulation of LKB1 in rat cortical progenitors with specific shRNAs using in utero electroporation [25], resulted in striking absence of axon formation in neurons in all cortical layers. Furthermore, because over-expressing LKB1 resulted in ectopic axon formation and branching [25], and because its deletion resulted in specific effect on axon formation from the basal pole, without affecting other aspects of neuronal development or migration $[22,25]$, we conclude that the level and localization of LKB1-activity need to be precisely regulated in the trailing process at the basal pole to ensure proper formation of the axon. Preferential activation of LKB1 in the unspecified trailing process may initiate axon development via local SAD phosphorylation and its downstream actions on Tau (see Fig. 5A), resulting in local MT polymerization that is necessary for axon formation [22, 23].

As the first step in the development of an in vivo technology for the localized introduction of intracellular proteins in cortical progenitors in live rodent embryos, we delivered chitosan micelles into developing rat cortical progenitors, in utero. After performing a C-section on E18 timed-pregnant rat, the uterine horns were exteriorized, and the embryos' cerebral hemispheres were exposed under the transparent uterine membrane (Fig. 7A). Chitosan micelles $(0.5 \mathrm{mg} / \mathrm{ml})$ suspended in PBS, were injected into one of the lateral ventricles, a potent neuronal stem cell niche, of E18 rat embryos through the uterine membrane. Based on the findings from cultured hippocampal neurons (Fig. 3 and 6), where we observed efficient uptake and prolonged cytoplasmic stability of chitosan-NCs, we predicted that micellar delivery into the lateral ventricles, will allow their uptake by a subset of cortical neuronal post-mitotic progenitors lining 
the ventricular wall, to allow their entry into the neuronal cytoplasm in vivo. Injection at E18 should mostly label layer II/III pyramidal cortical progenitors [37, 38] (Fig. 7A,B), whereby fluorescently-tagged chitosan micelles will allow tracking of these developing and migrating neurons in the embryo. Following the administration of micelles by injection, the embryos were placed back in the abdominal cavity. After allowing embryonic development in utero to continue for 2 days, embryonic cortical brain slices were obtained at E20, and high-resolution images of cortical neurons derived from chitosan-uptaken progenitor cells were acquired using Cy5.5 fluorescence. Our findings showed uptake of chitosan micelles in cortical progenitors, at the cortical plate (CP) (Fig. 7Ca-d) or radially migrating (Supplementary Fig. S5a,b), upon injection into lateral ventricles in utero, with chitosan present in the cytoplasm $2 \mathrm{~d}$ after injection. Furthermore, these results showed that chitosan uptake did not affect the survival or development of these neurons in vivo. These results demonstrated that we have developed a methodology for delivery of chitosan micelles into the live rodent embryonic cortex in utero (Fig. 7), to label newborn cortical progenitor cells.

\section{Discussion}

Fundamental events during embryonic neuronal development as neurogenesis, migration, directed formation of axon and dendrite, and synaptogenesis, are regulated by protein activity in a highly spatio-temporal manner. Our study aims to manipulate an early event in neuronal development, where neuronal cells polarize to create morphologically and functionally distinct compartments of axon and dendrite, a critical architecture that allows the directed connectivity and information flow in the brain. This polarized architecture is dictated by the ability of neuronal cells to spatially segregate the activity of specific intracellular proteins, including kinases, small 
GTPases, and actin and microtubule cytoskeleton regulators $[1-23,25]$. The failure in spatiotemporal protein compartmentalization may lead to aberrant polarization and, ultimately, to developmental neuropathologies, mental and motor disabilities, epilepsy, and autism spectrum disorders. Current approaches that are predominantly used for spatio-temporal manipulation of protein function, including genetic manipulations and light-controlled activation [27-30], are limited in their ability to sustain localized intracellular protein activity.

Our goal therefore is to establish a biomagnetic-based nanotechnology to deliver and retain localized activity of the intracellular kinase LKB1, to dictate axon formation in developing embryonic neurons in culture and in the live rodent embryonic brain in vivo. Here we took the initial steps toward the development of this methodology. We created a biomagnetic supramolecular assembly for effectively complexing with LKB1 to allow its rapid neuronal uptake and prolonged cytoplasmic availability and stability.

The LKB1 nanocomplex (LKB1-NC) consists of a polysaccharide-based micellar structure that is linked to magnetic nanoparticles (MNPs) via avidin-biotin binding. We expect that LKB1 delivery using the micellar nanocomplex will result in its availability in the cytoplasm upon rapid release from endocytic vesicles, where the proposed configuration will serve several crucial purposes. First, LKB1, a soluble kinase protein, will extend out from the micelle into the aqueous environment of the cytoplasm for optimal protein activity, while anchored locally by the MNPs (Fig. 1) and the magnetic field. Furthermore, because of the stable biotin-avidin linking and because the amphiphilic polymeric chains of chitosan will maintain a stable core-shell structure in the aqueous cytoplasm, our NC configuration with the linked LKB1 should allow prolonged localized LKB1 activity in the cytoplasm for up to 2 days, sufficient time for axon development, as was demonstrated (Fig. 6). Using a biochemical assay, we demonstrated that LKB1 linked to 
the nanocomplex retained its kinase activity and phosphorylated the downstream target Tau, in the presence of the effector SAD-A kinase (Fig. 5). The NCs were successfully delivered to cultured embryonic hippocampal neurons (Fig. 3 and 4). After delivery, the NCs were rapidly uptaken (within minutes), and not only appeared to be distributed within the cell soma, but were also present at the tip and throughout the length of the growing neuritic processes. There was clear colocalization between the components of the NC, chitosan, MNPs and fluorescein, for up to $2 \mathrm{~d}$, indicating $\mathrm{NC}$ integrity and stability in the cytoplasm for a prolonged time.

Nanoparticle capture within acidic intracellular vesicles following uptake constitutes the predominant barrier for the availability of protein activity in the cytoplasm. To allow rapid cellular uptake $[39,40]$ in neurons in culture (Fig. 3, 4, and 6) and in vivo (Fig. 7), and, more importantly, to mediate NCs endosomal-lysosomal escape $[39,40]$ and prolonged cytoplasmic stability following uptake, we chose to use chitosan-derived nanoparticles, because of their proposed ability to escape endocytic entrapment $[39,40]$. Our findings would support the choice of chitosan micelles as the scaffolding material for the NCs. The prolonged cytoplasmic availability and integrity of our designed NCs in the neuronal cytoplasm (Fig. 3 and 6) suggests that NCs are indeed stable in the cytoplasm long beyond endosomal half-life $(2-4 \mathrm{hr})[56,57]$, and that active LKB1 linked to the MNPs and anchored by the magnetic-field would be available in the cytoplasm. Direct examination of NCs endocytic routing, using a time-course analysis of chitosan micelle localization with early (EEA1) or late-lysosomal (LAMP1) endocytic markers, confirmed this premise. These results showed that although the nanoparticles were uptaken by the classical endocytic pathway, in which at early times they localized in early endosomes (data not shown), at no time did they localize within lysosomes (Supplementary Fig. S3). Together, the stability of the micelles in the neuronal cytoplasm for the 2-3 day period, indeed indicates their escape form 
lysosomal entrapment. These results showed that the facile chemical tailorability of chitosan indeed allowed its successful complexation with LKB1 and MNPs, for the rapid uptake and the prolonged stability and function of LKB1 in the neuronal cytoplasm.

The dimension of the chitosan micelle is important as it affects its uptake and intracellular routing. Furthermore, their size is a crucial consideration for localization in the fine neuritic processes, only few microns in diameter. Our synthesis scheme was designed to yield nanocomplexes with an average diameter of $200 \mathrm{~nm}$, as shown by dynamic light scattering (DLS) and transmission electron microscopy (TEM) data (Fig. 2). These dimensions should allow nanoparticle uptake via macropinocytosis [41, 42, 56, 57], a nonspecific and constitutive uptake process by which fluid contents are continuously taken up from the surrounding environment, thus representing an optimal route of uptake for our experiments. We note that our nanocomplexes ranged in size from $176 \mathrm{~nm}$ to $200 \mathrm{~nm}$ (Fig. 2). Several possible reasons might account for the final size of the composite NC when compared to the HGC micelle alone. First, the increased hydrophobic interaction between the micelle core and avidin, after binding with streptavidincoated MNP, might cause HGC to contract. Second, because the chitosan polymer was linked to the MNPs via strong but non-covalent binding, the resulting hybrid nanoparticle, LKB1-MNPHGC, would remain flexible, specifically due to the viscoelastic properties of chitosan. Lastly, after linking the streptavidin-coated MNPs to the chitosan micelles via avidin/biotin binding and prior to size measurement by dynamic light scattering, the nanocomplex mixture was briefly probe-sonicated, to homogenize the dispersion and yield a more monodisperse sample. During this step, the MNPs may have become encapsulated by the micelles, which were temporarily disrupted by the sonication, before their reassembly into an organic/inorganic hybrid nanoparticle [58]. 
These findings suggest that active LKB1 linked to and anchored by the MNPs will be available in the cytoplasm for $2 \mathrm{~d}$, sufficient time for axon formation. In support of this notion, we show that delivery of LKB1-linked micelles induced axon formation in these cultured hippocampal neurons (Fig. 6). Neurons that have uptaken the LKB1-micelles, efficiently formed multiple axons, unlike control neurons that formed only a single axon. Together with the biochemical examination of LKB1-activity in phosphorylating downstream targets (Fig. 5), the findings in cultured neurons show that LKB1 linked to chitosan NCs maintains its catalytic activity, and that it can efficiently activate the downstream signaling cascade in the neuronal cytoplasm, SAD-A and Tau phosphorylation, to promote axon formation. Therefore, we have provided the critical demonstration that the delivery of the LKB1 kinase would be sufficient in dictating axon formation. Together, these findings indicate that our platform is suitable for the localized delivery of LKB1 into a single undifferentiated neuritic process with the purpose of dictating its axonal fate.

To develop a nanotechnology for intracellular protein localized introduction in vivo, we first established a methodology for the delivery of the NCs into cortical progenitors in a live rat embryonic brain in utero. The previously developed in utero gene delivery methods to specific brain regions for gene up- or down-regulation $[37,38]$ have greatly advanced the morphological and functional examination of the developing brain, from the single cell to the developing network. Here, we performed in utero micellar delivery and uptake in a live rat embryo at E18. Rat embryonic lateral ventricles, a potent neuronal stem cell niche, were injected at E18 with chitosan micelles, and NCs uptake was examined two days post-injection at E20. We show that micellar delivery into the lateral ventricles, allowed their uptake by a subset of cortical neuronal postmitotic progenitors lining the ventricular wall, to allow their entry into the neuronal cytoplasm in 
vivo. These cortical progenitors expressed fluorescently-tagged chitosan micelles, and allowed the tracking of these developing and migrating neurons in the embryo. Confocal imaging analysis revealed that the micelles were indeed uptaken by cortical progenitor cells, radially migrating or at the cortical plate (Fig. 7), and stably remained in the cytoplasm for $2 \mathrm{~d}$ after injection. Intracellular micelle delivery into the live rodent embryonic brain, with the purpose of manipulating neuronal development, represents a unique approach, established in this study. Further development of local delivery and retention of LKB1-linked biomagnetic NCs in embryonic cortical progenitors in vivo, should allow the instruction of axon formation from the trailing process of these developing neurons, using an externally applied magnetic field.

Abolishment of cytoplasmic LKB1-activity underlies the developmental neuropathology Polyhydramnios, Megalencephaly and Symptomatic Epilepsy (PMSE), characterized by epilepsy and mental and motor disabilities [59,60], resulting in early childhood mortality. We hypothesize that PMSE is caused by defects in neuronal development, specifically, failure of axon formation. The nanotechnology developed in this study could be used for repair of axon development defects in cultured hippocampal neurons and in cortical progenitors in utero, in a mouse model for PMSE, by direct subcellular introduction and retention of LKB1-NCs.

Functionalized MNPs are used for imaging in biomedical research and clinical applications [61-63]. Few recent studies have used MNPs for the activation of cell signaling, however these studies were limited to signaling manipulations at the cell surface, by inducing cell-surface receptor clustering $[64,65]$, ion-channel activation [66], or ligand-receptor induced signaling endosome localization [67]. A more recent study used a magnetogenetic approach for spatial clustering of Rho-GTPases to control local cytoskeleton remodeling in cultured fibroblasts [68], however, this study was limited to manipulations for short time periods (min). Therefore, 
functionalized MNPs, though widely used, have not been applied toward long-term developmental events. Our use of MNPs is fundamentally different from these previous studies, as our nanocomplexes are designed for prolonged cytoplasmic stability of the intracellular protein kinase LKB1, sustained for up to $48 \mathrm{hr}$, both in rodent cultured neurons and in the embryonic cortex in vivo, to manipulate the complex developmental process of axon formation.

In this work we demonstrated that our nanotechnology is feasible in a single cultured neuronal cell and an intact rat embryonic brain in utero. The developed delivery methodology allowed the manipulation of a complex developmental process, axon formation, over a physiologically relevant time scale, demonstrating the sufficiency of intracellular protein function in dictating a central developmental event. Together, these studies constitute a critical proof of concept for the suitability of the engineered biomagnetic micellar system as a protein delivery platform for neuronal cells, to manipulate long-term developmental events that are dictated by localized intracellular protein function.

\section{Conclusions}

We here report the development of a micellar biomagnetic system that can be used to restrict the activity of LKB1, a critical intracellular kinase for neuronal polarization, in a spatiotemporal manner, to regulate axon development, in cultured neurons and in the live rodent embryonic brain in utero. LKB1 was complexed with amphiphilic chitosan micelles and MNPs, yielding a stable engineered nanocomplex that preserved kinase-activity. Our findings showed that the nanocomplexes were rapidly uptaken by cultured hippocampal neurons without affecting neuronal viability or development, and LKB1-chitosan induced axon formation in these cultured neurons. Lastly, we present a nanotechnology for in utero delivery and uptake of chitosan micelles 
in cortical progenitors in live rat embryonic brain in utero. Our findings establish chitosan micelles as a suitable scaffolding material, for subcellular delivery of critical intracellular proteins that can be manipulated upon complexing with magnetic nanoparticles, to dictate central events in neuronal development in vitro and in the live rodent embryonic brain. This study is applicable for the delivery and retention of many intracellular proteins, for assessing proper localization and timing of protein function in a wide variety of central and peripheral neurons. Our methodology can ultimately lead to the generation of new protein-based therapeutic approaches for the repair of neuropathologies that result from break-down of polarity.

\section{Acknowledgements}

This project was supported by a grant from NIH, NS084111 (M. Shelly), and the SUNY M\&AM Network of Excellence grant (Y. Meng). Research was carried out in part at the Center for Functional Nanomaterials, Brookhaven National Laboratory, which is supported by the U.S. Department of Energy, Office of Basic Energy Sciences, under Contract No. DE-SC0012704. We thank J. Cathcart for help with the endocytic routing studies.

\section{References}

[1] Arimura N, Kaibuchi K. Neuronal polarity: from extracellular signals to intracellular mechanisms. Nat Rev Neurosci. 2007;8:194-205. (10.1038/nrn2056)

[2] Dotti CG, Sullivan CA, Banker GA. The establishment of polarity by hippocampal-neurons in culture. J Neurosci. 1988;8:1454-68.

[3] Dotti CG, Banker GA. Experimentally induced alteration in the polarity of developing neurons. Nature. 1987;330:254-6. (10.1038/330254a0) 
[4] Barnes AP, Solecki D, Polleux F. New sights into the molecular mechanisms specifying neuronal polarity in vivo. Curr Opin Neurobiol. 2008;18:44-52. (10.1016/j.conb.2008.05.003)

[5] Barnes AP, Polleux F. Establishment of Axon-Dendrite Polarity in Developing Neurons. Annual Review of Neuroscience. 2009;32:347-81. (10.1146/annurev.neuro.31.060407.125536)

[6] Hirokawa N, Niwa S, Tanaka Y. Molecular Motors in Neurons: Transport Mechanisms and Roles in Brain Function, Development, and Disease. Neuron. 2010;68:610-38. (10.1016/j.neuron.2010.09.039)

[7] Witte H, Bradke F. The role of the cytoskeleton during neuronal polarization. Curr Opin Neurobiol. 2008;18:479-87. (10.1016/j.conb.2008.09.019)

[8] Shi SH, Jan LY, Jan YN. Hippocampal neuronal polarity specified by spatially localized mPar3/mPar6 and PI 3-kinase activity. Cell. 2003;112:63-75. (10.1016/s0092-8674(02)01249-7)

[9] Yoshimura T, Kawano Y, Arimura N, Kawabata S, Kikuchi A, Kaibuchi K. GSK-3 beta regulates phosphorylation of CRMP-2 and neuronal polarity. Cell. 2005;120:137-49. (10.1016/j.cell.2004.11.012)

[10] Jiang H, Guo W, Liang XH, Rao Y. Both the establishment and the maintenance of neuronal polarity require active mechanisms: Critical roles of GSK-3 beta and its upstream regulators. Cell. 2005;120:123-35. (10.1016/j.cell.2004.12.033)

[11] Toriyama M, Shimada T, Kim KB, Mitsuba M, Nomura E, Katsuta K, et al. Shootin1: a protein involved in the organization of an asymmetric signal for neuronal polarization. J Cell Biol. 2006;175:147-57. (10.1038/jcb.200404160)

[12] de Anda FC, Pollarolo G, Da Silva JS, Camoletto PG, Feiguin F, Dotti CG. Centrosome localization determines neuronal polarity. Nature. 2005;436:704-8. (10.1038/nature03811) 
[13] Da Silva JS, Hasegawa T, Miyagi T, Dotti CG, Abad-Rodriguez J. Asymmetric membrane ganglioside sialidase activity specifies axonal fate. Nature Neuroscience. 2005;8:606-15. $(10.1038 / \mathrm{nn} 1442)$

[14] Inagaki N, Chihara K, Arimura N, Menager C, Kawano Y, Matsuo N, et al. CRMP-2 induces axons in cultured hippocampal neurons. Nature Neuroscience. 2001;4:781-2. (10.1038/90476)

[15] Schwamborn JC, Puschel AW. The sequential activity of the GTPases Rap1B and Cdc42 determines neuronal polarity. Nature Neuroscience. 2004;7:923-9. (10.1038/nn1295)

[16] Jacobson C, Schnapp B, Banker GA. A change in the selective translocation of the Kinesin-1 motor domain marks the initial specification of the axon. Neuron. 2006;49:797-804. (10.1016/j.neuron.2006.02.005)

[17] Chen YM, Wang QJ, Hu HS, Yu PC, Zhu J, Drewes G, et al. Microtubule affinity-regulating kinase 2 functions downstream of the PAR-3/PAR-6/atypical PKC complex in regulating hippocampal neuronal polarity. Proc Natl Acad Sci U S A. 2006;103:8534-9. (10.1073/pnas.0509955103)

[18] Cheng PL, Lu H, Shelly M, Gao H, Poo MM. Phosphorylation of E3 ligase Smurf1 switches its substrate preference in support of axon development. Neuron. 2011;69:231-43. (10.1016/j.neuron.2010.12.021)

[19] Fivaz M, Bandara S, Inoue T, Meyer T. Robust neuronal symmetry breaking by Ras-triggered local positive feedback. Curr Biol. 2008;18:44-50. (10.1016/j.cub.2007.11.051)

[20] Shelly M, Lim BK, Cancedda L, Heilshorn SC, Gao H, Poo MM. Local and long-range reciprocal regulation of cAMP and cGMP in axon/dendrite formation. Science. 2010;327:547-52. $(10.1126 /$ science.1179735) 
[21] Cheng P-L, Song A-H, Wong Y-H, Wang S, Zhang X, Poo M-M. Self-amplifying autocrine actions of BDNF in axon development. Proc Natl Acad Sci U S A. 2011;108:18430-5. (10.1073/pnas.1115907108)

[22] Barnes AP, Lilley BN, Pan YA, Plummer LJ, Powell AW, Raines AN, et al. LKB1 and SAD kinases define a pathway required for the polarization of cortical neurons. Cell. 2007;129:549-63. (10.1016/j.cell.2007.03.025)

[23] Kishi M, Pan YA, Crump JG, Sanes JR. Mammalian SAD kinases are required for neuronal polarization. Science. 2005;307:929-32. (10.1126/science.1107403)

[24] Bony G, Szczurkowska J, Tamagno I, Shelly M, Contestabile A, Cancedda L. Nonhyperpolarizing $\mathrm{GABA}(\mathrm{B})$ receptor activation regulates neuronal migration and neurite growth and specification by cAMP/LKB1. Nature Communications. 2013;4. (10.1038/ncomms2820)

[25] Shelly M, Cancedda L, Heilshorn S, Sumbre G, Poo MM. LKB1/STRAD promotes axon initiation during neuronal polarization. Cell. 2007;129:565-77. (10.1016/j.cell.2007.04.012)

[26] Shelly M, Poo MM. Role of LKB1-SAD/MARK pathway in neuronal polarization. Dev Neurobiol. 2011;71:508-27. (10.1002/dneu.20884)

[27] Wu YI, Frey D, Lungu OI, Jaehrig A, Schlichting I, Kuhlman B, et al. A genetically encoded photoactivatable Rac controls the motility of living cells. Nature. 2009;461:104-U11. $(10.1038 /$ nature08241)

[28] Deiters A. Light activation as a method of regulating and studying gene expression. Curr Opin Chem Biol. 2009;13:678-86. (10.1016/j.cbpa.2009.09.026)

[29] Levskaya A, Weiner OD, Lim WA, Voigt CA. Spatiotemporal control of cell signalling using a light-switchable protein interaction. Nature. 2009;461:997-1001. (10.1038/nature08446) 
[30] Umeda N, Ueno T, Pohlmeyer C, Nagano T, Inoue T. A Photocleavable Rapamycin Conjugate for Spatiotemporal Control of Small GTPase Activity. J Am Chem Soc. 2011;133:124. $(10.1021 / \mathrm{ja} 108258 \mathrm{~d})$

[31] St Johnston D, Ahringer J. Cell Polarity in Eggs and Epithelia: Parallels and Diversity. Cell. 2010;141:757-74. (10.1016/j.cell.2010.05.011)

[32] Watts JL, Morton DG, Bestman J, Kemphues KJ. The C-elegans par-4 gene encodes a putative serine-threonine kinase required for establishing embryonic asymmetry. Development. $2000 ; 127: 1467-75$.

[33] Spicer J, Rayter S, Young N, Elliott R, Ashworth A, Smith D. Regulation of the Wnt signalling component PAR1A by the Peutz-Jeghers syndrome kinase LKB1. Oncogene. 2003;22:4752-6. (10.1038/sj.onc.1206669)

[34] Lizcano JM, Goransson O, Toth R, Deak M, Morrice NA, Boudeau J, et al. LKB1 is a master kinase that activates 13 kinases of the AMPK subfamily, including MARK/PAR-1. Embo Journal. 2004;23:833-43. (10.1038/sj.emboj.7600110)

[35] Biernat J, Wu YZ, Timm T, Zheng-Fischhofer QY, Mandelkow E, Meijer L, et al. Protein kinase MARK/PAR-1 is required for neurite outgrowth and establishment of neuronal polarity. Mol Biol Cell. 2002;13:4013-28. (10.1091/mbc.02.03.0046)

[36] Chin A, Suarato G, Meng Y. Evaluation of physicochemical characteristics of hydrophobically modified glycol chitosan nanoparticles and their biocompatibility in murine osteosarcoma and osteoblast-like cells. Journal of Nanotechnology and Smart Materials 2014;1:17. 
[37] Noctor SC, Martinez-Cerdeno V, Ivic L, Kriegstein AR. Cortical neurons arise in symmetric and asymmetric division zones and migrate through specific phases. Nat Neurosci. 2004;7:13644. $(10.1038 / \mathrm{nn} 1172)$

[38] Saito T, Nakatsuji N. Efficient gene transfer into the embryonic mouse brain using in vivo electroporation. Dev Biol. 2001;240:237-46. (10.1006/dbio.2001.0439)

[39] Deli MA. Potential use of tight junction modulators to reversibly open membranous barriers and improve drug delivery. Biochim Biophys Acta-Biomembr. 2009;1788:892-910. (10.1016/j.bbamem.2008.09.016)

[40] Malatesta M, Galimberti V, Cisterna B, Costanzo M, Biggiogera M, Zancanaro C. Chitosan nanoparticles are efficient carriers for delivering biodegradable drugs to neuronal cells. Histochem Cell Biol. 2014;141:551-8. (10.1007/s00418-013-1175-9)

[41] Conner SD, Schmid SL. Regulated portals of entry into the cell. Nature. 2003;422:37-44. $(10.1038 /$ nature01451)

[42] Duncan R, Richardson SCW. Endocytosis and Intracellular Trafficking as Gateways for Nanomedicine Delivery: Opportunities and Challenges. Mol Pharm. 2012;9:2380-402. $(10.1021 / \mathrm{mp} 300293 n)$

[43] Morille M, Passirani C, Vonarbourg A, Clavreul A, Benoit JP. Progress in developing cationic vectors for non-viral systemic gene therapy against cancer. Biomaterials. 2008;29:3477-96. (10.1016/j.biomaterials.2008.04.036)

[44] Park JH, Kwon SG, Nam JO, Park RW, Chung H, Seo SB, et al. Self-assembled nanoparticles based on glycol chitosan bearing 5 beta-cholanic acid for RGD peptide delivery. Journal of Controlled Release. 2004;95:579-88. (10.1016/j.jconrel.2003.12.020) 
[45] Green NM. Avidin .3. Nature of biotin-binding site. Biochem J. 1963;89:599-\&. $(10.1042 / b j 0890599)$

[46] Green NM. Avidin .1. Use of 14C biotin for kinetic studies and for assay. Biochem J. 1963;89:585-\&. (10.1042/bj0890585)

[47] Pacini S, Vannelli GB, Barni T, Ruggiero M, Sardi I, Pacini P, et al. Effect of 0.2 T static magnetic field on human neurons: remodeling and inhibition of signal transduction without genome instability. Neuroscience Letters. 1999;267:185-8. (10.1016/s0304-3940(99)00362-6)

[48] Baas AF, Boudeau J, Sapkota GP, Smit L, Medema R, Morrice NA, et al. Activation of the tumour suppressor kinase LKB1 by the STE20-like pseudokinase STRAD. EMBO J. 2003;22:3062-72. (10.1093/emboj/cdg292)

[49] Baas AF, Kuipers J, van der Wel NN, Batlle E, Koerten HK, Peters PJ, et al. Complete polarization of single intestinal epithelial cells upon activation of LKB1 by STRAD. Cell. 2004;116:457-66. (10.1016/S0092-8674(04)00114-X)

[50] Rakic P. Neuron-glia relationship during granule cell migration in developing cerebellar cortex. A Golgi and electronmicroscopic study in Macacus Rhesus. J Comp Neurol. 1971;141:283312. $(10.1002 /$ cne. 901410303$)$

[51] Rakic P. Mode of cell migration to the superficial layers of fetal monkey neocortex. J Comp Neurol. 1972;145:61-83. (10.1002/cne.901450105)

[52] Shoukimas GM, Hinds JW. The development of the cerebral cortex in the embryonic mouse: an electron microscopic serial section analysis. J Comp Neurol. 1978;179:795-830. $(10.1002 /$ cne.901790407)

[53] Gao WQ, Hatten ME. Neuronal differentiation rescued by implantation of Weaver granule cell precursors into wild-type cerebellar cortex. Science. 1993;260:367-9. 
[54] Komuro H, Yacubova E, Yacubova E, Rakic P. Mode and tempo of tangential cell migration in the cerebellar external granular layer. J Neurosci. 2001;21:527-40.

[55] Hatanaka Y, Murakami F. In vitro analysis of the origin, migratory behavior, and maturation of cortical pyramidal cells. J Comp Neurol. 2002;454:1-14. (10.1002/cne.10421)

[56] Doherty GJ, McMahon HT. Mechanisms of Endocytosis. Annu Rev Biochem. Palo Alto: Annual Reviews; 2009. p. 857-902.

[57] Petros RA, DeSimone JM. Strategies in the design of nanoparticles for therapeutic applications. Nat Rev Drug Discov. 2010;9:615-27. (10.1038/nrd2591)

[58] Du JZ, Sun H. Polymer/TiO2 Hybrid Vesicles for Excellent UV Screening and Effective Encapsulation of Antioxidant Agents. Acs Applied Materials \& Interfaces. 2014;6:13535-41. $(10.1021 / \mathrm{am} 502663 \mathrm{j})$

[59] Puffenberger EG, Strauss KA, Ramsey KE, Craig DW, Stephan DA, Robinson DL, et al. Polyhydramnios, megalencephaty and symptomatic epilepsy caused by a homozygous 7-kilobase deletion in LYK5. Brain. 2007;130:1929-41. (10.1093/brain/awm100)

[60] Orlova KA, Parker WE, Heuer GG, Tsai V, Yoon J, Baybis M, et al. STRAD alpha deficiency results in aberrant mTORC1 signaling during corticogenesis in humans and mice. J Clin Invest. 2010;120:1591-602. (10.1172/jci41592)

[61] Pankhurst QA, Connolly J, Jones SK, Dobson J. Applications of magnetic nanoparticles in biomedicine. Journal of Physics D-Applied Physics. 2003;36:R167-R81. (10.1088/0022$3727 / 36 / 13 / 201)$

[62] Petters C, Dringen R. Accumulation of iron oxide nanoparticles by cultured primary neurons. Neurochemistry International. 2015;81:1-9. (10.1016/j.neuint.2014.12.005) 
[63] Pilar Calatayud M, Riggio C, Raffa V, Sanz B, Torres TE, Ricardo Ibarra M, et al. Neuronal cells loaded with PEI-coated Fe3O4 nanoparticles for magnetically guided nerve regeneration. Journal of Materials Chemistry B. 2013;1:3607-16. (10.1039/c3tb20336k)

[64] Mannix RJ, Kumar S, Cassiola F, Montoya-Zavala M, Feinstein E, Prentiss M, et al. Nanomagnetic actuation of receptor-mediated signal transduction. Nat Nanotechnol. 2008;3:3640. (10.1038/nnano.2007.418)

[65] Cho MH, Lee EJ, Son M, Lee J-H, Yoo D, Kim J-w, et al. A magnetic switch for the control of cell death signalling in in vitro and in vivo systems. Nat Mater. 2012;11:1038-43. $(10.1038 /$ nmat3430)

[66] Huang H, Delikanli S, Zeng H, Ferkey DM, Pralle A. Remote control of ion channels and neurons through magnetic-field heating of nanoparticles. Nat Nanotechnol. 2010;5:602-6. $(10.1038 /$ nnano.2010.125)

[67] Steketee MB, Moysidis SN, Jin XL, Weinstein JE, Pita-Thomas W, Raju HB, et al. Nanoparticle-mediated signaling endosome localization regulates growth cone motility and neurite growth. Proc Natl Acad Sci U S A. 2011;108:19042-7. (10.1073/pnas.1019624108)

[68] Etoc F, Lisse D, Bellaiche Y, Piehler J, Coppey M, Dahan M. Subcellular control Rac-GTPase signalling by magnetogenetic manipulation inside living cells. Nat Nanotechnol. 2013;8:193-8. $(10.1038 /$ nnano.2013.23) 


\section{Figure Legends}

Fig. 1. Schematic representation of the synthesis of hydrophobically modified glycol chitosan (HGC) and formation of LKB1-chitosan and LKB1-MNP-HGC nanocomplexes

(Step 1) Sulfo-NHS-LC-biotin is conjugated with glycol chitosan (GC) to form biotinylated glycol chitosan (BGC); (Step 2) BGC is allowed to bind with avidin to form avidinylated BGC (AB-GC); (Step 3) A self-assembled micelle is formed upon conjugation of 5 $\beta$-cholanic acid with either ABGC (3a) or BGC (3b). (Step 4) Prior to the complexation of LKB1 to the micelle, AB-HGC is labeled with Cy5.5 to form Cy5.5-AB-HGC (4a) and B-HGC is linked to streptavidin-coated magnetic nanoparticles (MNP) to yield B-HGC-MNP (4b); (Step 5) Sulfo-NHS-LC-biotin is coupled with LKB1 to form biotinylated-LKB1, which then binds to the avidinylated moieties on either Cy5.5-AB-HGC (Step 6a) or B-HGC-MNP (Step 6b). As a result, the Cy5.5-labeled LKB1 chitosan and the LKB1-MNP-HGC nanocomplexes are formed. Table (top, right) shows the biotin:chitosan molar ratio before (BGC) and after (AB-GC) the avidinylation of glycol chitosan. Immunoblotting (bottom, left) of HEK-293T cell lysates expressing Flag-tagged rat LKB1, compared to lysates from untransfected cells (-), was performed using LKB1-Ab. "Total lysate", total cell lysates. The LKB1 was purified from the total cell-lysate using Flag-Ab, and 1\% or 2.5\% of the total purified LKB1 used for linking to the nanocomplex, was also immunoblotted.

\section{Fig. 2. Nanoparticle size and morphology}

Dynamic light scattering data and transmission electron micrographs of (A) hydrophobically modified glycol chitosan (HGC), (B) $100 \mathrm{~nm}$ magnetic nanoparticles (MNP), (C) biotinylatedHGC-MNP (B-HGC-MNP), (D) Cy5.5-labeled LKB1-chitosan nanocomplex, and (E) LKB1MNP-HGC nanocomplex. Scale bar, $300 \mathrm{~nm}$ (A), $200 \mathrm{~nm}(\mathrm{~B}, \mathrm{D}, \mathrm{E}), 100 \mathrm{~nm}(\mathrm{C})$. The data indicate 
that chitosan micelles and MNPs were $200 \mathrm{~nm}$ and $100 \mathrm{~nm}$ in diameter, respectively, and the complexing of the two materials yielded a particle of $\sim 170 \mathrm{~nm}$ in size. LKB1-chitosan nanocomplexes, both with and without MNP, were $\sim 180 \mathrm{~nm}$ in diameter.

\section{Fig. 3. Delivery of chitosan micelles, MNPs, or triple-component NCs into cultured hippocampal neurons}

(A) Schematic presentation of axon and dendrite formation during neuronal polarization in dissociated cultured rodent embryonic hippocampal neurons. The polarization process has been divided into five stages. Shortly after culturing, the neuron forms small membranous protrusions (stage 1) that develop into short and morphologically similar neurites $20-30 \mu \mathrm{m}$ in length within 6-12 $\mathrm{hr}$ (stage 2). Apparent polarity first emerges after $24 \mathrm{hr}$ (stage 3), when one of the neurites exhibits accelerated growth and acquires axonal characteristics. Maturation of axon and dendrites occurs in subsequent days (stage 4), followed by synapse formation (stage 5 ).

(B,C,D) Confocal microscopy images of cultured E18 rat hippocampal neurons that have uptaken the following nanocomplexes: (B) Cy5.5-labeled hydrophobically modified glycol chitosan (HGC) micelles, (C) $413 \mathrm{~nm}$ fluorophore labeled magnetic nanoparticles (MNPs), or (D) the triplecomponent NC, consisting of Cy5.5-labeled chitosan micelles complexed with biotinylatedfluorescein and MNPs. The cultures were applied with the nanocomplexes at $0.125-0.5 \mathrm{mg} / \mathrm{ml}$, and incubated for 18,30 , or $48 \mathrm{hr}$. The NCs were uptaken by the neurons and were present in cytoplasm and neuritic processes (arrows), at the tip of growing neuritic processes or along the neurites, for up to $48 \mathrm{hr}$. In the triple-component $\mathrm{NC}$, clear co-localization was found between chitosan, MNPs and fluorescein (D), indicating NCs cytoplasmic integrity and stability for $30 \mathrm{hr}$. Scale bar, $30 \mu \mathrm{m}$. 
(E,F) Composite tracing of neurites from a random sampling of hippocampal neurons $(\mathrm{n}=10)$ from each treatment, as a measure of neurite length and number. Neurites were traced from the randomly selected cells and overlaid to generate the composite image. Shown are traces of neurons that were applied with: chitosan micelles (E) for 18 or $24 \mathrm{hr}$; Triple-component NCs (F) consisting of chitosan micelles complexed with fluorescein and MNPs, for $30 \mathrm{hr}$. Control cultures were left untreated. Scale bar, $30 \mu \mathrm{m}$.

Fig. 4. Quantitative measurements of neurite length and number upon delivery of chitosan micelles or triple-component NCs into cultured hippocampal neurons

$(\mathbf{A}, \mathbf{B})$ Quantitation of average $( \pm \mathrm{SE})$ individual neurite length for the first three longest neurites

for each cell, average of total neurite length per cell, and average of individual neurite length per cell ( $n=2-3$ cultures, 30-60 cells), for neurons that were applied with: chitosan micelles (A) for 18 or $24 \mathrm{hr}$; Triple-component NCs (B) consisting of chitosan micelles complexed with biotinylated-fluorescein and MNPs, for $30 \mathrm{hr}$. Control cultures were left untreated.

(C,D) Quantitation of average ( \pm SE) neurite number for each cell $(\mathrm{n}=2-3$ cultures, 30-60 cells), for neurons that were applied with: chitosan micelles (C) for 18 or $24 \mathrm{hr}$; Triple-component NCs (D) for $30 \mathrm{hr}$, for same data set as in (A,B). Control cultures were left untreated.

Fig. 5. LKB1-linked to chitosan micelles maintained catalytic activity in phosphorylating downstream targets

(A) Schematic depiction of LKB1-signaling cascade in axon formation. Activation of LKB1 leads to activation and phosphorylation of SAD-A/B kinases that subsequently phosphorylate the MT- 
associated protein Tau on the serine residue 262, necessary events in LKB1-mediated axon formation.

(B) Immunoblots of HeLa cell-lysates transfected with cDNA for Tau, either alone or together with cDNAs for SAD-A or the LKB1-cofactor STRAD $\alpha$, incubated with LKB1-linked or control chitosan micelles for $2 \mathrm{hr}$, prior to gel-electrophoresis. Tau phosphorylation was detected by immunoblotting with phospho-site-specific Ab at Ser262, and compared between LKB1- or control-chitosan-micelle treated lysates. Immunoblotting with non-phosphorylated Tau Ab served control for Tau expression. LKB1-linked chitosan micelles phosphorylated Tau in lysates that coexpressed SAD-A and STRAD $\alpha$ together with Tau (red arrowhead). Phosphorylation of Tau by LKB1-chitosan micelles was comparable to that of control lysates from cells in which LKB1cDNA was co-expressed together with Tau, SAD-A and STRAD $\alpha$ (on the right). Immunoblotting for SAD-A and STRAD $\alpha$ confirmed their expression in the respective lysates. Immunoblotting for $\beta$-actin served control for protein loading.

Fig. 6. LKB1-linked chitosan micelles induced multiple axon formation in cultured hippocampal neurons

(A) Example images of hippocampal neurons bath-applied with either LKB1-linked or controlchitosan micelles, fixed at $60 \mathrm{hr}$, and immunostained with the axon marker Smi-312 together with the neuronal marker Tuj-1. Tracings of the neurons, including axons (red) and dendrites (green), determined based on Smi-312 labeling, are presented at the bottom. Scale bar, $25 \mu \mathrm{m}$. LKB1micelles induced formation of multiple axons in many neurons, whereas in cultures applied with control micelles, most neurons formed only one axon. 
(B) Cell tracings of representative neurons, including soma, axon (red), and dendrites (green), from a random sampling of hippocampal neurons ( $\mathrm{n}=10)$, applied with LKB1-linked or control-chitosan micelles. Scale bar, $50 \mu \mathrm{m}$. Many cells in cultures applied with LKB1-micelles formed multiple axons (arrows), unlike control neurons that mostly formed only one axon.

(C,D) Quantitative measurements of percentage of cells $( \pm$ SE) forming either single or multiple axons (C), or multiple axons only (D), in LKB1-linked or control-chitosan micelle applied cultures, demonstrating considerable increase in multiple axon formation upon LKB1-micelles application compared to control. The data show 35-60 cells each, from 2 separate cultures.

(E) LKB1-linked Cy5.5-labeled chitosan was present in the soma of hippocampal neurons at 60 hr. Representative image of a hippocampal neuron that formed multiple axons upon application of LKB1-chitosan micelles, immunostained with Smi-312 and Tuj-1, at $60 \mathrm{hr}$. Scale bar, $50 \mu \mathrm{m}$.

(F) Super-Resolution Structured Illumination Microscopy (SIM) reconstructed Z-stack image of hippocampal neurons applied with LKB1-linked Cy5.5-labeled chitosan micelles, immunostained at $60 \mathrm{hr}$ for the lysosomal marker LAMP1, demonstrating that chitosan micelles did not co-localize with lysosomes. Scale bar, $5 \mu \mathrm{m}$.

Fig. 7. Delivery of chitosan micelles into cortical progenitors in a live rat embryonic brain in utero

(A) Schematic depiction of the in utero chitosan-micelle delivery procedure. After performing a C-section on E18 timed-pregnant rat, the uterine horns were exteriorized, and the embryos' brain cerebral hemispheres were exposed. Cy5.5-Chitosan micelles $(0.5 \mathrm{mg} / \mathrm{ml})$ were injected into one of the lateral ventricles of E18 rat embryos, to label layer II/III pyramidal neuronal progenitors. After allowing embryonic development in utero to continue for 2 days, embryonic cortical brain 
slices were obtained at E20, and high-resolution confocal microscopy images of cortical neurons derived from chitosan-uptaken progenitors were acquired using Cy5.5 fluorescence.

(B) Schematic representation of cortical neuronal development. An embryonic cortical brain slice, is shown on the left, depicting the lateral ventricles injected with chitosan micelles. On the right, shown is the stereotypical development of cortical progenitors. Upon completion of mitosis, cortical progenitors start to migrate radially towards the cortical plate $(\mathrm{CP})$ to populate the different layers of the developing cortex. During migration, these neurons undergo stereotypical morphological changes leading to polarized development of axon and dendrite. The newborn neuron acquires a bipolar morphology in the ventricular zone (VZ), with apical-leading and basaltrailing processes. The trailing process will become the axon and will grow towards its target. On the right, depicted are the different cortical regions: "VZ / SVZ", ventricular zone / sub-ventricular zone; "IZ", intermediate zone; "CP", cortical plate.

(C) Confocal microscopy images of E20 rat cortical progenitors injected in utero at E18 with Cy5.5-chitosan micelles $(0.5 \mathrm{mg} / \mathrm{ml})$, counterstained with DAPI for cortical layer determination. $(b, c)$ and (d) show the boxed regions in (a), and (c), respectively, representing higher magnification images of cortical neurons derived from chitosan-uptaken progenitor cells, acquired using Cy5.5 fluorescence. Chitosan micelles are present in the cytoplasm of neurons at the CP (a-d), $2 \mathrm{~d}$ after injection. Scale bar, $200 \mu \mathrm{m}$ (a), $100 \mu \mathrm{m}(\mathrm{b}, \mathrm{c}), 20 \mu \mathrm{m}$ (d). Total of $3 \sim 5$ embryonic brains were analyzed from 2 separate timed-pregnant rats. Chitosan uptake did not affect the survival or development of these cortical neurons in vivo. 
Fig. 1

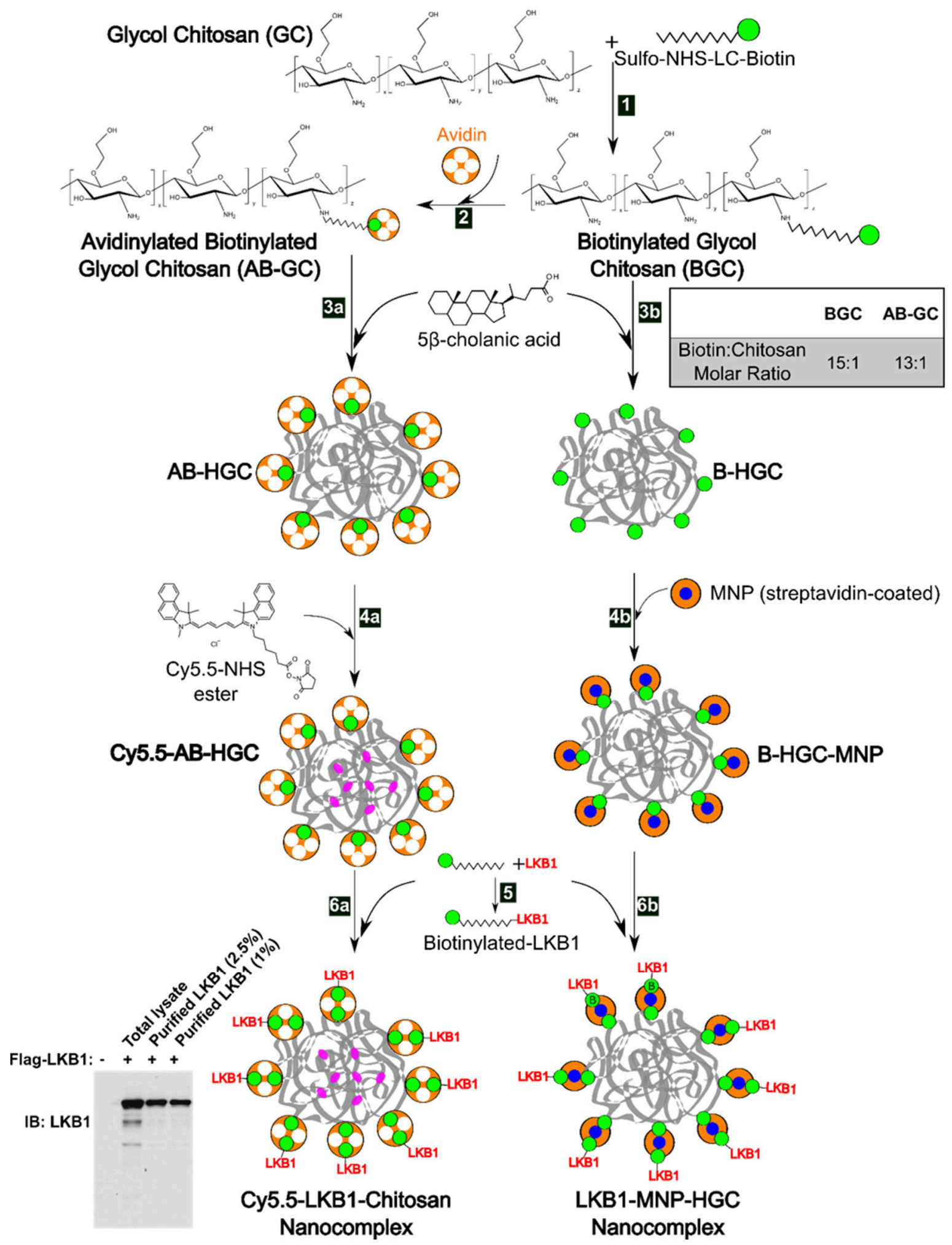


Fig. 2

(A)
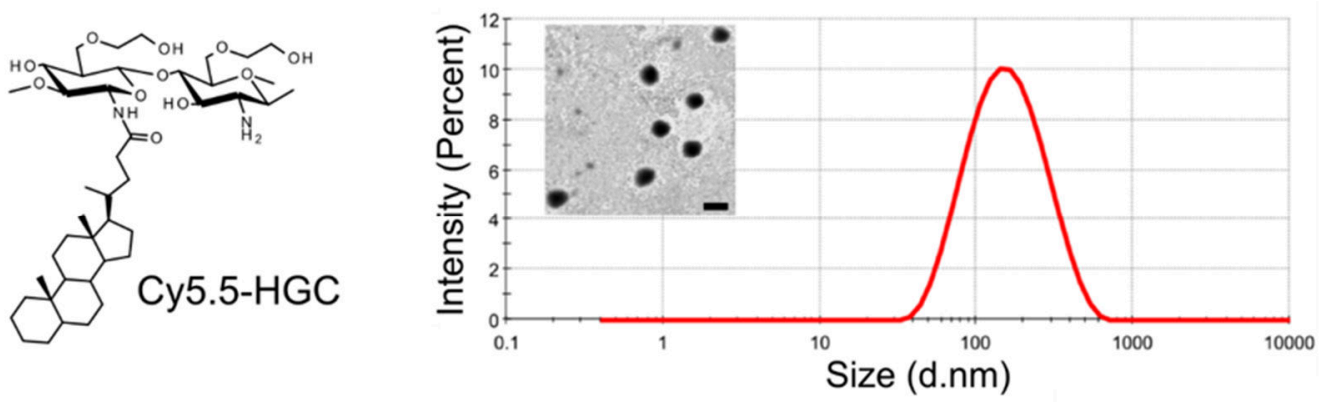

(B)

(C)

Streptavidin-coated MNP
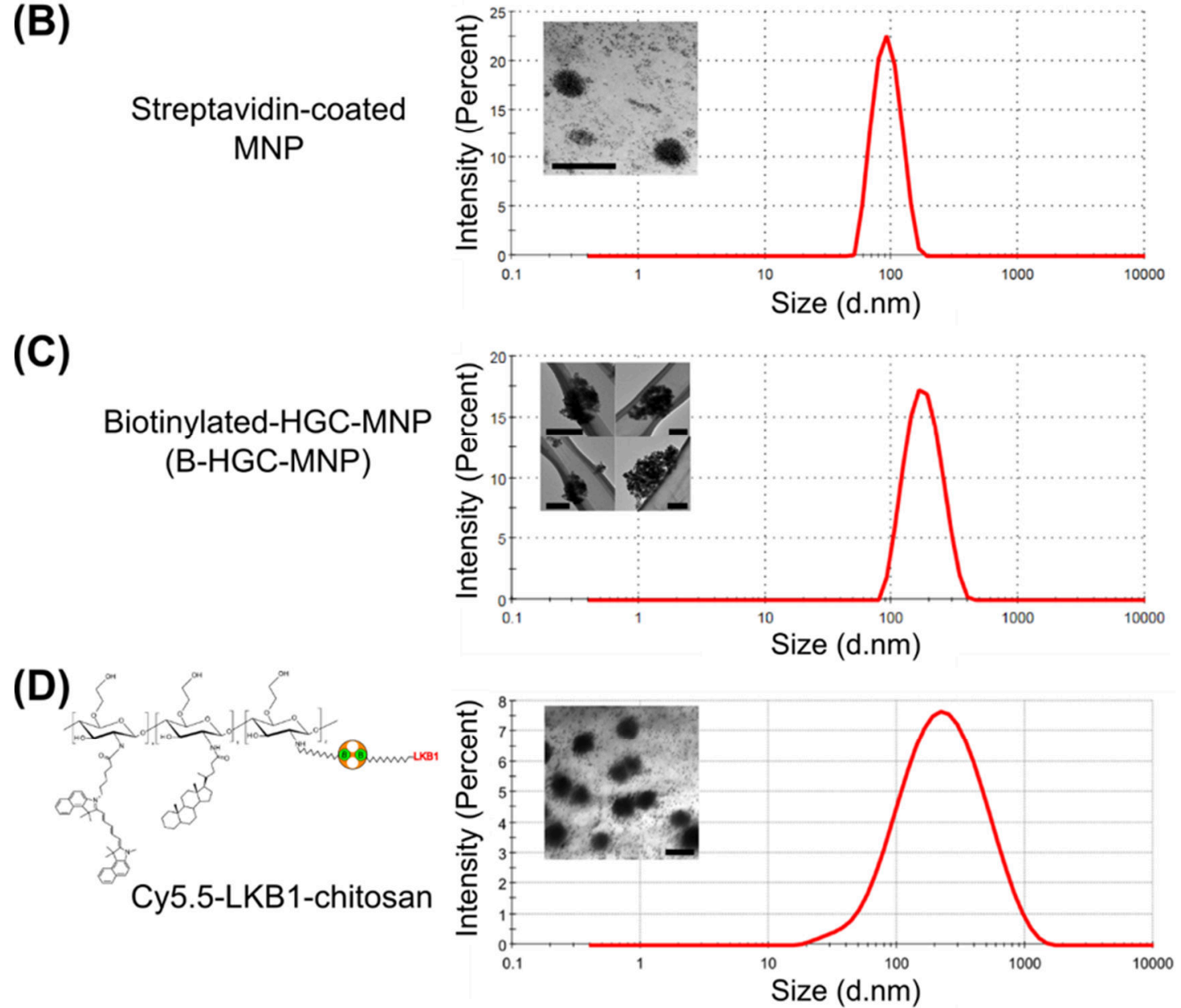

(E)
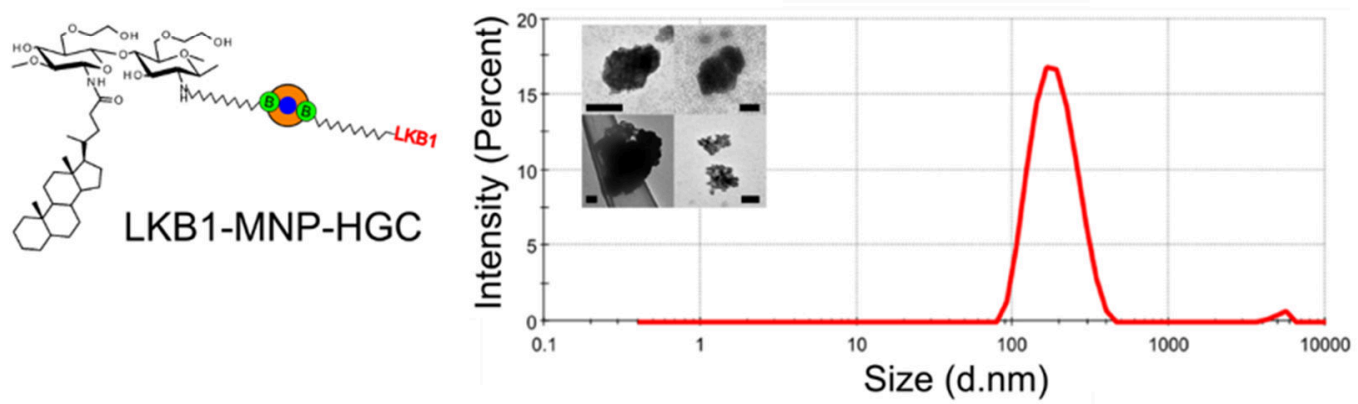
Fig. 3

(A) Cultured rodent hippocampal neurons

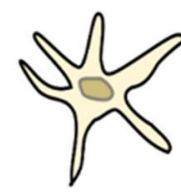

Stage 1

(first 6 hr)

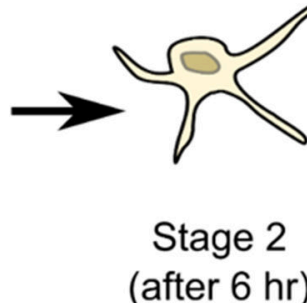

(after 6 hr)
(B)

(D)
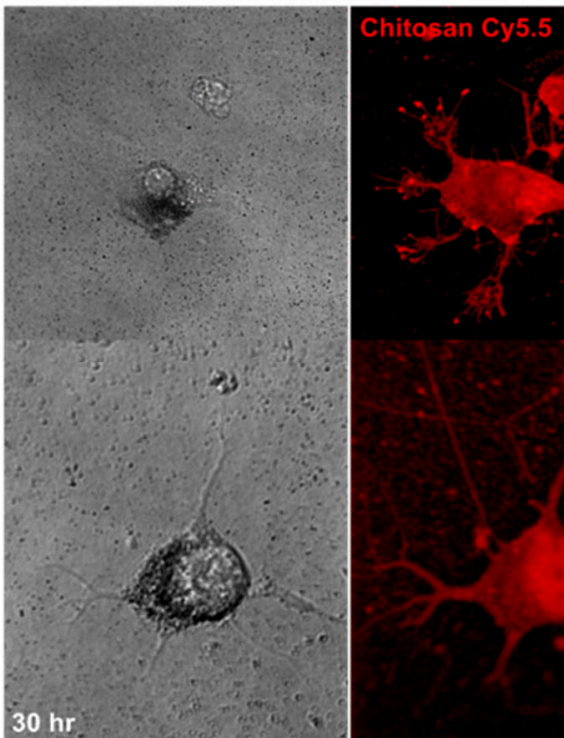

(E)

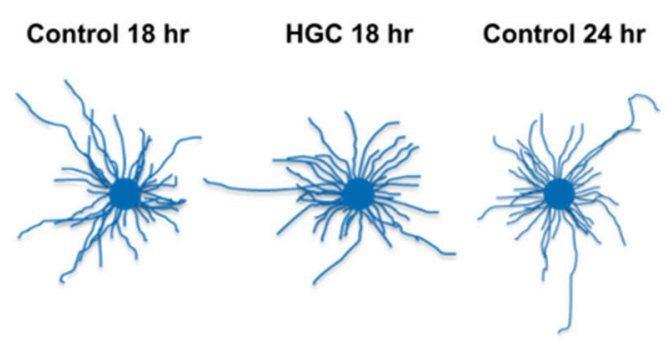

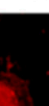
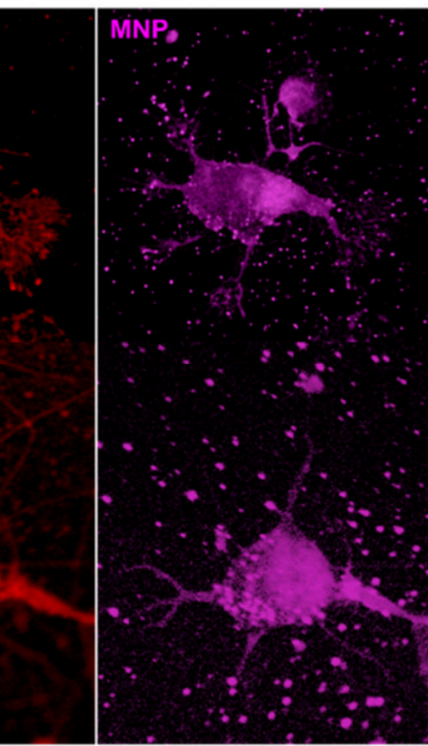

Biotinylated Fluorescein

(C)
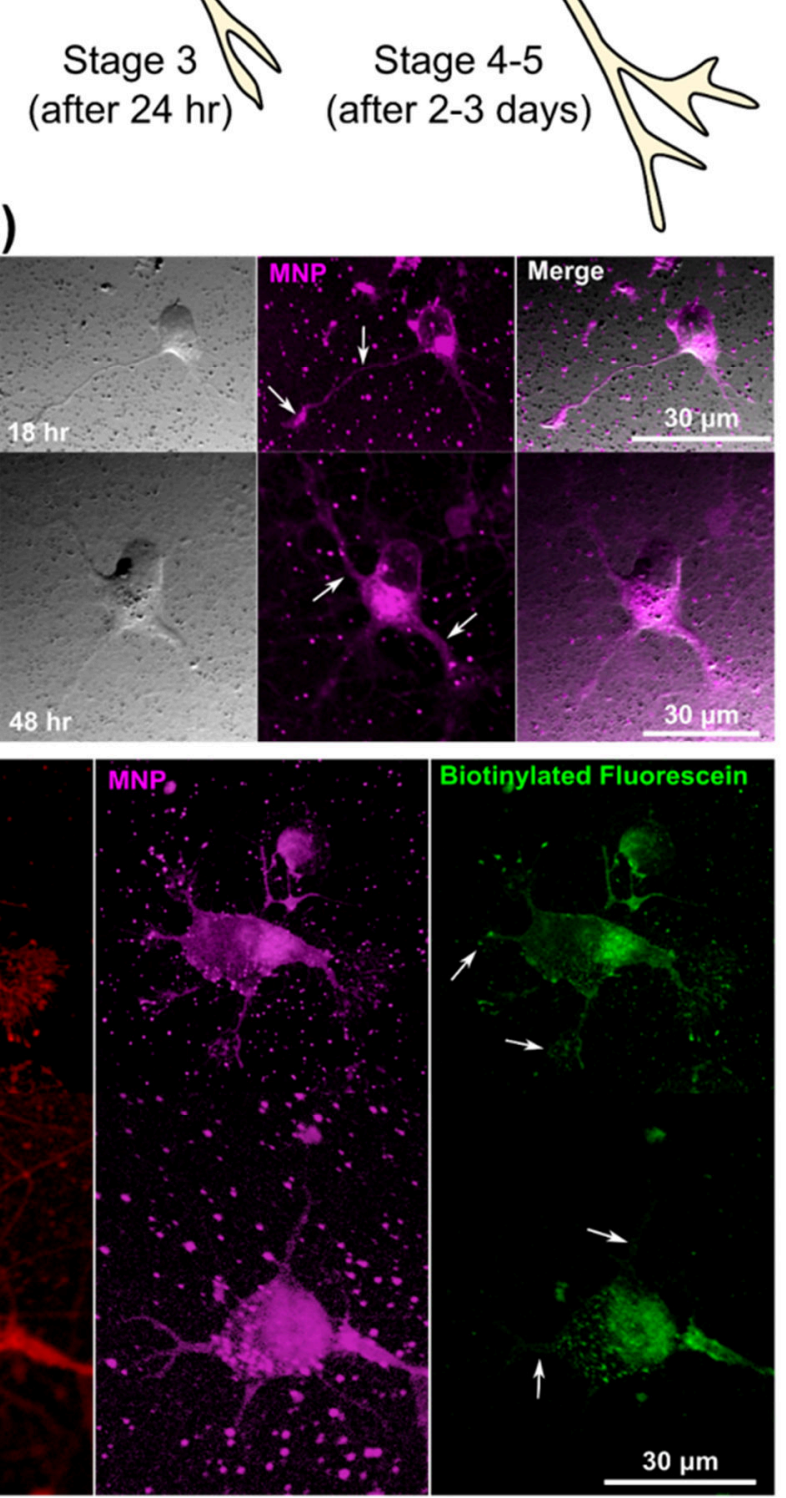

HGC 24 hr

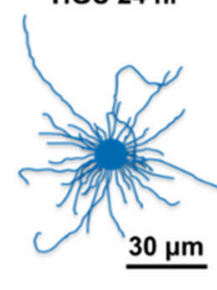

(F) Control $30 \mathrm{hr}$ Triple-component NC $30 \mathrm{hr}$ 
Fig. 4

(A)

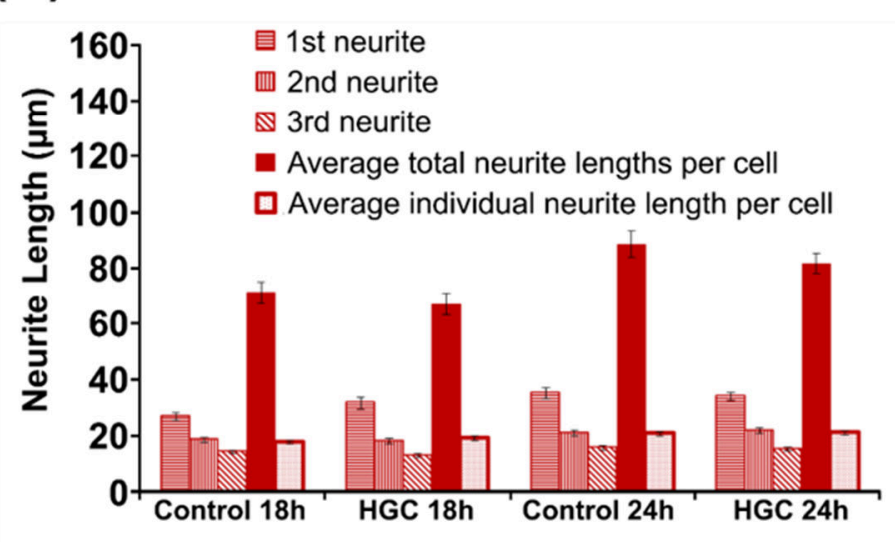

(C)

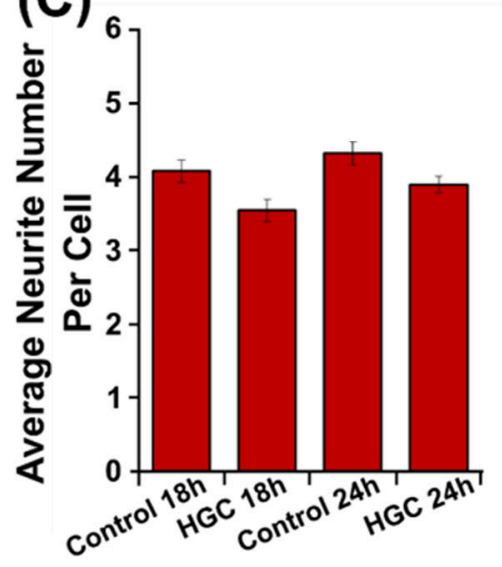

\section{Triple-Component NC}

(B)

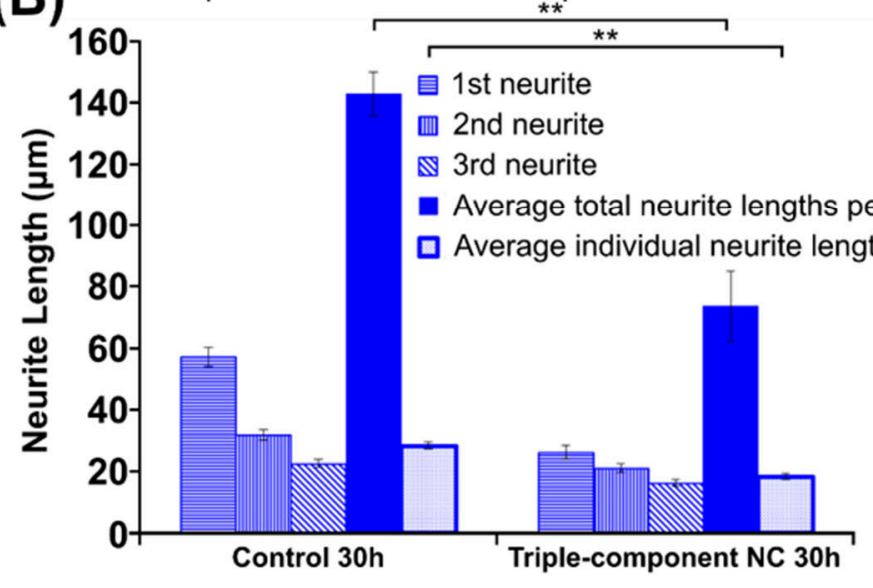

(D)

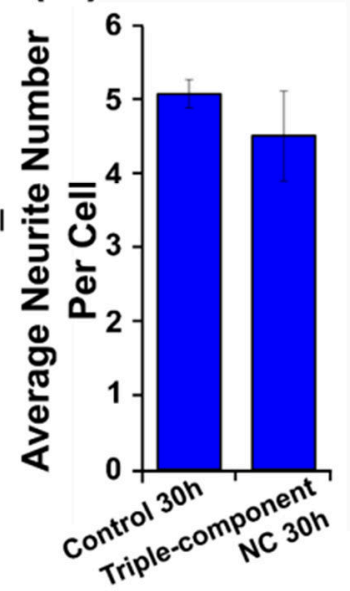


Fig. 5

(A)

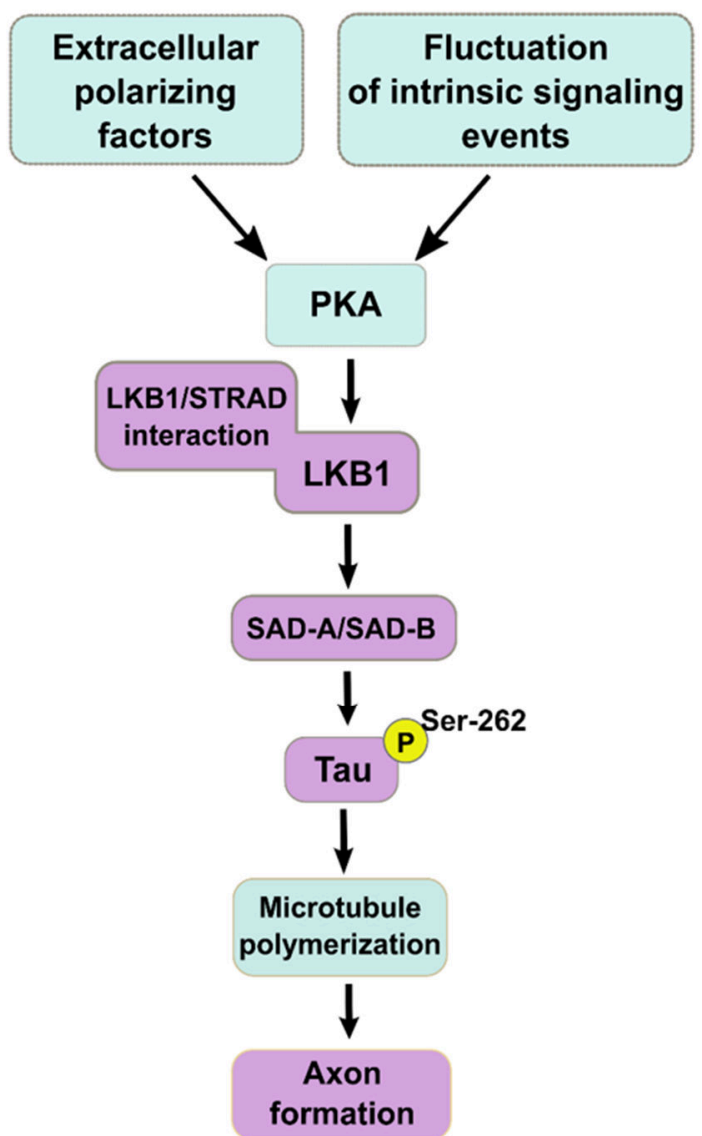

(B)

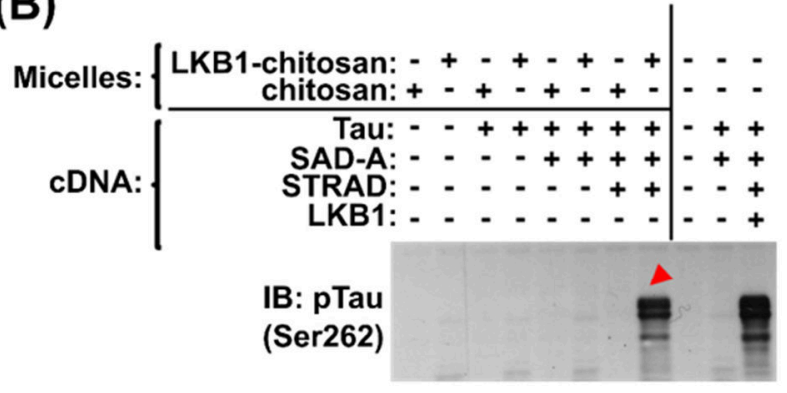

IB: Tau

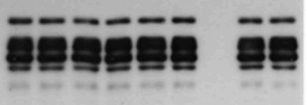

IB: SAD-A

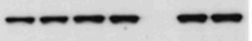

IB: STRAD

IB: LKB1

IB: $\beta$-actin 
(A)
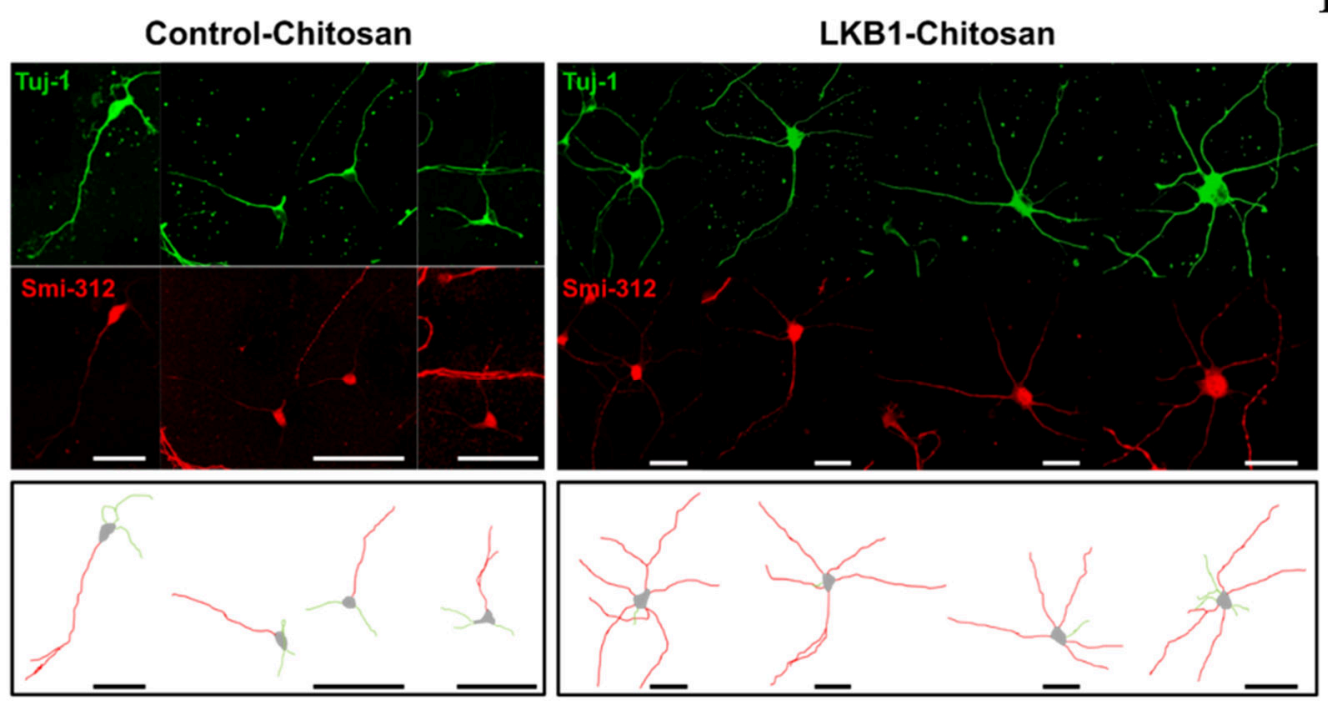

(B)

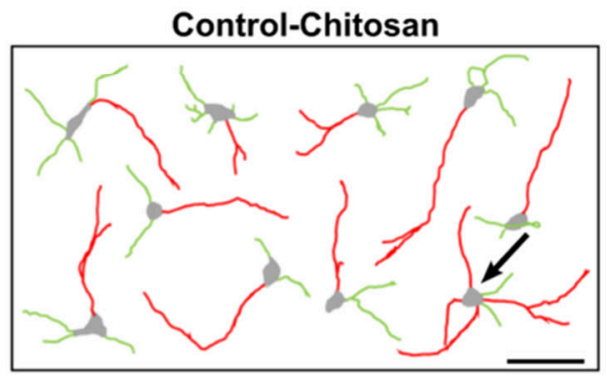

(C)

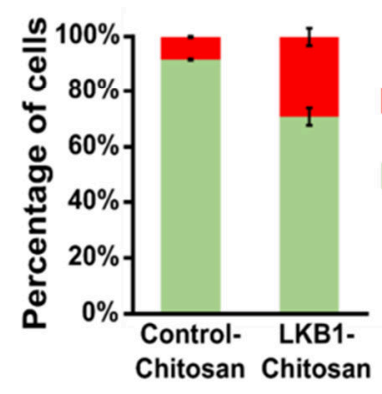

(E)

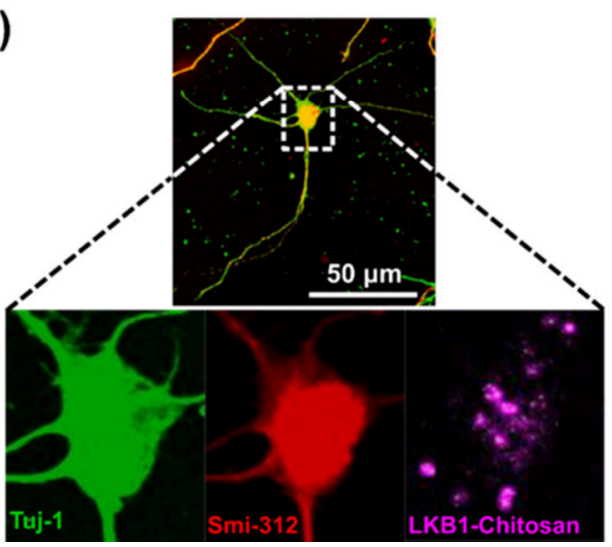

Fig. 6
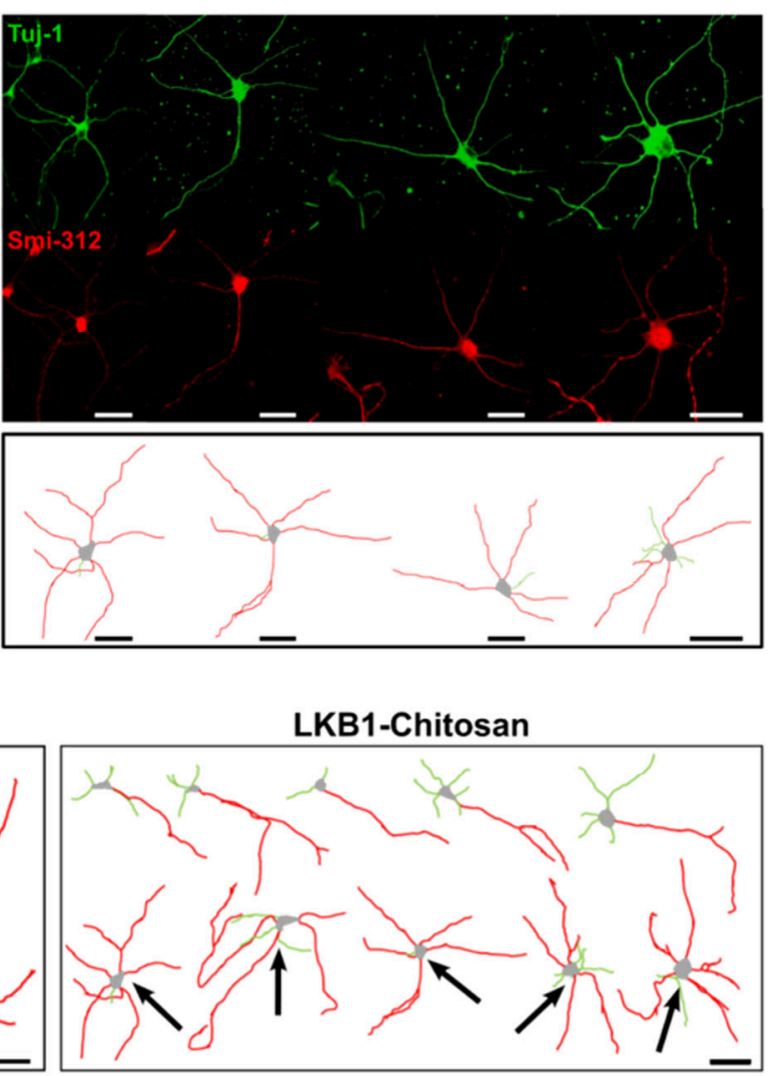

(D)

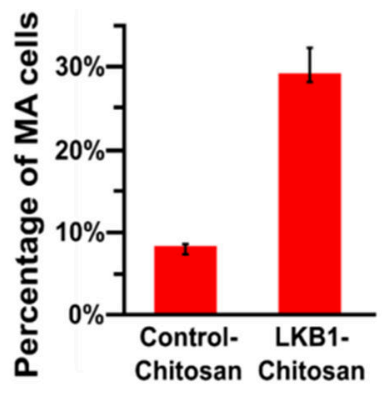

(F)

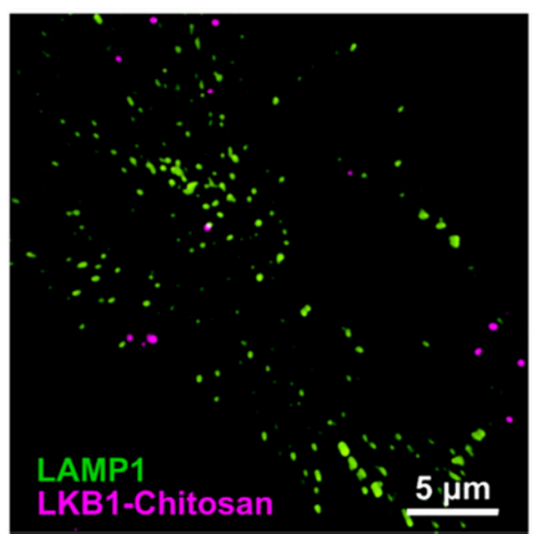


Fig. 7

(A)

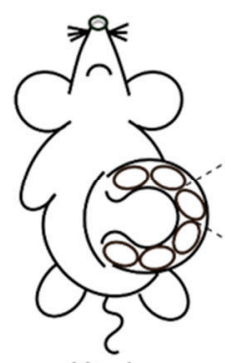

Uterine

horns

exposure

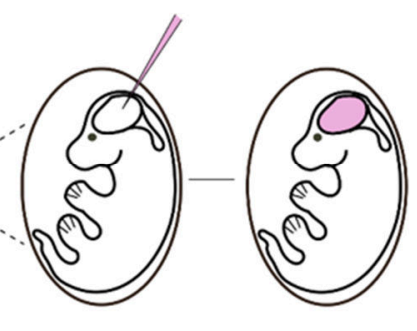

Chitosan micelle injection

Chitosan micelles in the

ventricles

(B) Top view

(C)

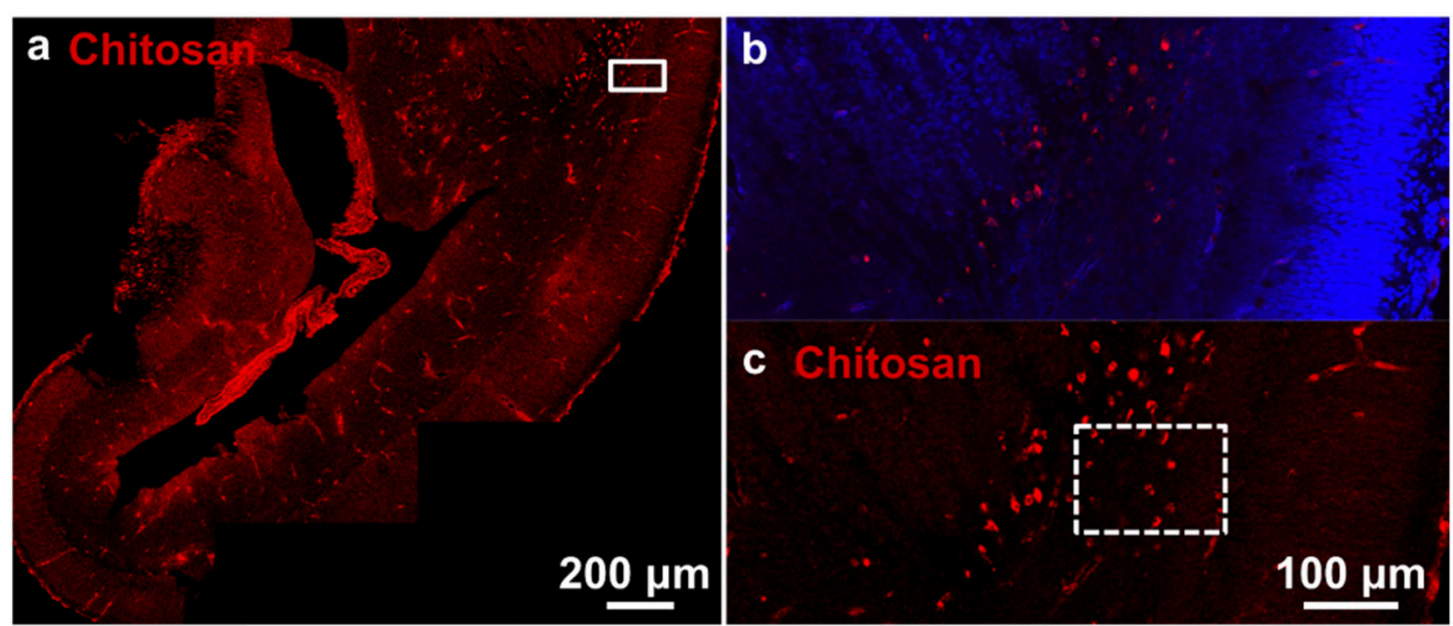

d Chitosan

$20 \mu \mathrm{m}$ 